\title{
The FluxMax Approach: Simultaneous Flux Optimization and Heat Integration by Discretization of Thermodynamic State Space Illustrated on Methanol Synthesis Process
}

\author{
Dominik Schack ${ }^{\mathrm{a}}$, Georg Liesche ${ }^{\mathrm{a}}$, Kai Sundmacher ${ }^{\mathrm{a}, \mathrm{b}}$ \\ ${ }^{a}$ Max Planck Institute for Dynamics of Complex Technical Systems, Department Process Systems \\ Engineering, Sandtorstr.1, D-39106 Magdeburg, Germany \\ ${ }^{b}$ Otto-von-Guericke-University Magdeburg, Department Process Systems Engineering, Universitätsplatz 2, \\ D-39106 Magdeburg, Germany
}

\begin{abstract}
Resource efficiency is a key driver in the chemical industry for both economic and ecological reasons. However, often the design of chemical processes or units and the corresponding heat integration, is divided into two design phases: a flow optimization to identify an optimal design and the subsequent evaluation of the heat integration potential. This procedure cannot guarantee the identification of the global resource optimum, which increases the need for a method that can do both simultaneously. This is the aim of the FluxMax approach that discretizes the thermodynamic state space. The introduction of nodes corresponding to mixtures, elementary processes and utilities allows the representation of any chemical process as a directed graph, which decouples effectively process-based nonlinearities from the optimization problem. Heat integration is considered by additional constraints. Using the methanol synthesis process as example, energy-optimal process configurations are identified that outperform configurations identified in a sequential procedure.
\end{abstract}

Keywords: Integrated Process Design, Process Optimization, Energy Efficiency, Methanol Synthesis, Heat Integration

\section{1. Introduction}

In the context of energy transition, one of the major goals of the chemical industry is to 3 substitute fossil feedstock with sustainable technologies and the use of renewable resources.

4 But even if the focus is on the substitution of feedstock, an increase in efficiency is crucial for Preprint submitted to Chemical Engineering Science

November 5, 2019 
a successful transition to a more sustainable production of chemicals (International Energy Agency, 2018). In order to enhance the overall process efficiency, challenges must be faced at different levels of detail. While at the production system level, more general questions and early stage decisions of chemical production networks are addressed (Otto et al., 2015, Voll and Marquardt, 2012, Schack et al., 2018), at the plant level, the aim is the identification of optimal process configurations, that consists of single process units, such as reactors, separators, and heat exchangers (Ulonska et al., 2016, Huang et al., 2018, Uebbing et al., 2019). In contrast, at the process level the performance of single units, such as reactor or separator, is in the focus (Kaiser et al., 2017, Keßler et al., 2019).

For the design of process systems, mixed integer formulation are often used to account for the binary decision whether an alternative is active or not (Hartono et al., 2012, Voll and Marquardt, 2012, Short et al., 2018). However, there are also optimization based approaches that avoid binary decision variables. Kim et al. (2013) analyzed optimal strategies for converting biomass into fuels. A (continuous) linear programming (LP) formulation was derived by considering the yield as a parameter, which led to a linear dependency on the production capacity. In contrast, Schack et al. (2018) introduced continuous process extent variables to avoid binary decision variables.

While in many bio-based applications, heat integration is often not of key interest, as the temperatures are too low (Zondervan et al., 2011, Voll and Marquardt, 2012, Kim et al., 2013), in most publications, heat integration and corresponding energy reduction potentials are in the main focus. In general, there are two different approaches to consider heat integration within optimization based methods: in a sequential procedure the flow optimization is solved first and subsequently a Pinch-based analysis is performed to evaluate the heat integration potential (Kokossis et al., 2015, Ulonska et al., 2016, Gençer and Agrawal, 2018). Also the utilization of excess heat to generate electricity is in the focus of recent publications (Yu et al., 2017, Elsido et al., 2017, Kermani et al., 2018). The advantage of a sequential procedure is that the complexity of the optimization is usually decreased because no additional constraints have to be considered to account for the heat integration. However, the sequential procedure does not ensure the identification of energy-optimal processes. 
To guarantee the identification of the mass- and energy optimum a simultaneous procedure has to be followed, in which the heat integration is an integrated part of the flow optimization problem (Papoulias and Grossmann, 1983a,b, Duran and Grossmann, 1986). The idea of the model proposed by Duran and Grossmann (1986) is to consider all feasible and non-feasible pinch combinations within the optimization problem and to identify the feasible pinch by maximization of the total utility requirements. As the number of additional constraints grows rapidly for complex systems, which makes the solution of the MINLP problem impossible, the model was further developed, e.g. by splitting the heat flows into dedicated zones, in which heat integration is allowed (Colberg and Morari, 1990, Yee et al., 1990, Dowling and Biegler, 2015, Huang et al., 2018). The increasing complexity is also the limiting factor in the p-graph approach of Friedler et al. (1992). They ended up with over 10,000 possible heat exchangers in their MILP formulation of a relative simple superstructure of a single reactor and three separation stages (Nagy et al., 2001).

The infinitely dimensional state space framework (IDEAS), which was first proposed by Wilson and Manousiouthakis (2000), might help to overcome the challenges of a large complexity, as they iteratively solve a linear program. In this way, the global optimum is approximated by increasing the dimension at each iteration. Later, heat integration was also included in their studies (Holiastos and Manousiouthakis, 2002, 2004) by pre-selecting possible heat transferring streams.

The methodology of Elementary Process Functions (EPF) was proposed by Freund and Sundmacher (2008). In a multi-step approach, a matter element was tracked in the thermodynamic state space and an optimal trajectory was calculated. In a final step, the optimal trajectory was used as the basis for the invention of a real process. The methodology was successfully applied to catalytic gas phase reactions (Peschel et al., 2010) and multiphase reactors (Hentschel et al., 2014, Kaiser et al., 2017). Recent developments based on this methodology also enable the design of so-called tolerant chemical reactors capable of processing multiple raw materials (Maußner et al., 2019).

In the present work, we propose the FluxMax approach for the simultaneous mass flux optimization and heat integration of chemical processes across different length scales by dis- 
cretization of the thermodynamic state space. The focus is on the detailed illustration of the key features of the FluxMax approach, such as the network representation of arbitrary chemical processes and the heat integration model, which enables the identification of optimal process and unit designs, depending on the considered length-scale. The introduction of four different types of nodes allows the representation of the chemical process as a directed graph, with the edges corresponding to the mass and energy fluxes to be optimized. The FluxMax approach follows three steps: i) discretization of the thermodynamic state space, ii) modeling the elementary processes, that characterize the transformation between the discrete state points, and iii) formulation and solution of the flux optimization problem. The discretization of the thermodynamic state space effectively decouples process-based nonlinearities from the network flow problem, resulting in a linear feasible region. In this way, the conceptual methodology of the linear programming approach (Schack et al., 2016, 2018) and the decoupling strategy applied in (Liesche et al., 2018) can be used as base of the proposed approach. By adding additional inequality constraints, heat integration is considered as integrated part of the flux optimization.

The FluxMax approach is applied to the methanol synthesis process, which is of great significance for applications in the field of Renewable-to-Chemicals (R2Chem). In particular the usage as storage molecule of so-called surplus energy raises interest, because methanol $\left(\mathrm{CH}_{3} \mathrm{OH}\right)$ is liquid at ambient temperature. Surplus energy is used to produce hydrogen $\left(\mathrm{H}_{2}\right)$ via electrolysis, which is then converted in a reaction with carbon dioxide $\left(\mathrm{CO}_{2}\right)$ into methanol. Thus besides beneficial storage properties, the consumption of $\left(\mathrm{CO}_{2}\right)$ explains the high significance of methanol in R2Chem applications.

Energy-optimal process configurations are identified by using a linear objective function: minimizing the total energy requirement. The influence of the considered discretization of the thermodynamic state space is discussed, as the optimal solution depends strongly on the considered grid. Using an appropriate discretization, it will be shown that the FluxMax approach identifies energy-optimal process configurations that outperform those identified in a sequential procedures, underscoring the importance of a simultaneous approach. Furthermore, the possibility of optimizing the utility network and the corresponding temperature 
levels will be demonstrated.

\section{The FluxMax approach}

The general idea of the FluxMax approach (FMA) is an effective decoupling of processbased nonlinearities from the subsequent network flux optimization by discretization of the thermodynamic state space. The discretization allows the representation of chemical process across different lengthscales, which enables the transformation of a nonlinear process optimization problem into a convex flux optimization on a defined network graph. The FluxMax approach can be divided into three steps - i) discretization of the thermodynamic state space; ii) modeling of elementary process functions; and iii) formulation and solution of the flux optimization problem - that are illustrated in Fig. 1.

The first step comprises the discretization of the thermodynamic state space into thermodynamic state points (Fig. 1 (top)). In this way, it is possible to calculate the nonlinear thermodynamic potentials, such as enthalpy and entropy, a priori. In a second steps, the discrete state points are connected by elementary process functions as shown in Fig. 1 (center). Depending on the type of design application, these elementary process functions represent micro changes in the thermodynamic state space that are combined into process units or fully engineered process units such as distillation columns, reactors and other apparatuses. Thus, the FluxMax approach is applicable to different levels of complexity: production system, plant, or even process level. Characteristic quantities of the elementary process functions, such as specific energy demands, can be calculated a priori, because the linked thermodynamic state points are defined a priori. Thereby, the nonlinear preprocessing is fully decoupled from the third step: the network flux optimization under simultaneous consideration of heat integration (Fig. 1 (bottom)). The feasible region of the flux optimization is linear in terms of the fluxes that are decision variables.

The FluxMax approach - or previous work on which the FluxMax approach is based on - has been successfully applied to different levels of the chemical process hierarchy: at the i) production system level for the systematic analysis of different feedstock and energy sources of the methanol (Schack et al., 2018) and formic acid production process (Schack 


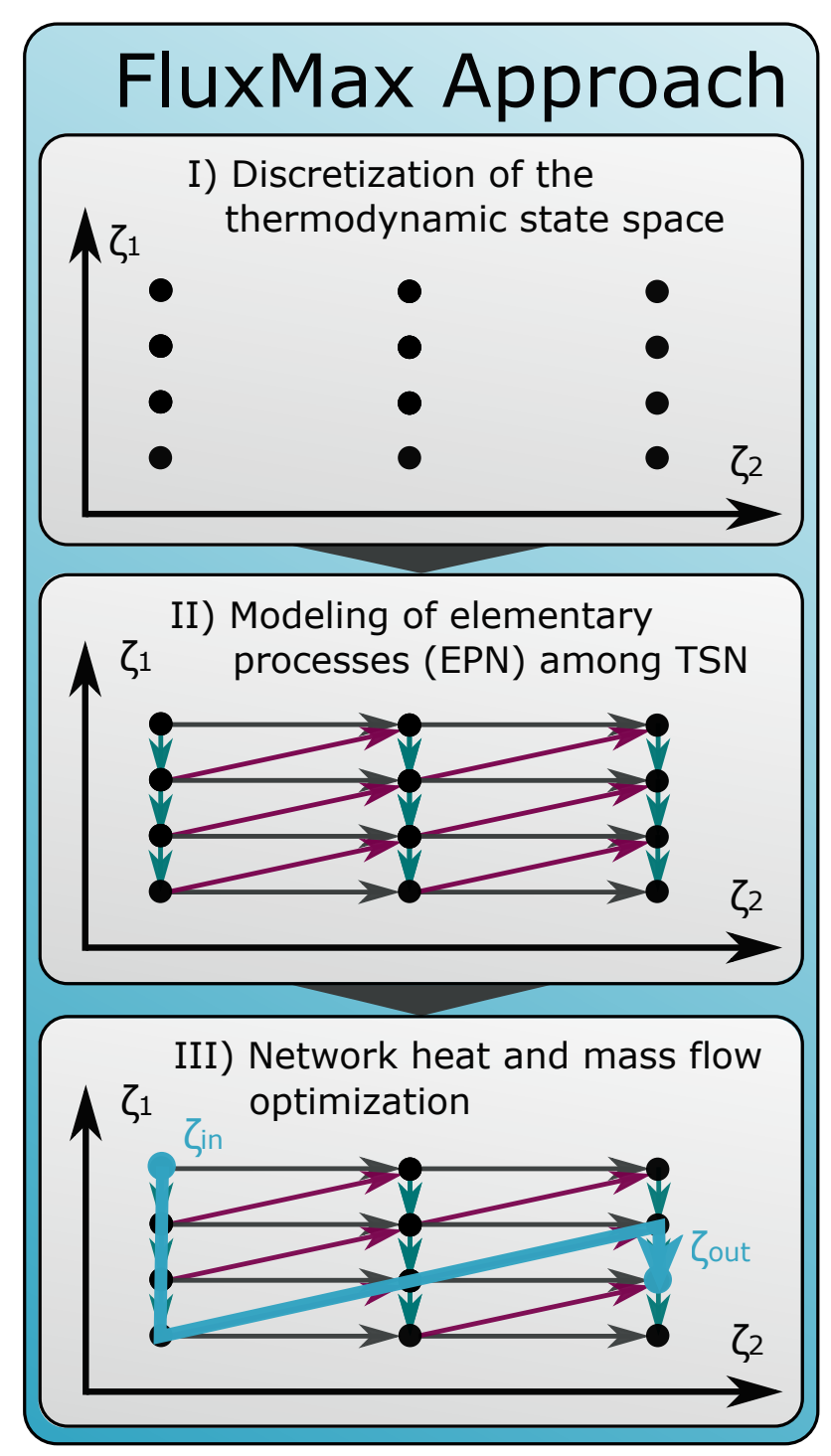

Figure 1: Illustration of the three-step FluxMax Approach for unit and process design with simultaneous heat integration: discretization of thermodynamic state space (I), modeling of elementary processes (II), and formulation and solution of the flow optimization (III); the thermodynamic state space is spanned by its thermodynamic coordinates $\zeta_{z}$, where $z$ denotes the number of dimension. 
and Sundmacher, 2018), ii) plant level to compare reactor designs of the energy-intensive hydrogen cyanide process (Liesche et al., 2019), and iii) process level to optimize the compressor cascade and the reactor part (Liesche et al., 2018) as well as the separation part of the methanol synthesis process (Schack et al., 2019).

In literature the linearization of the feasible region was also applied previously. Within the IDEAS framework, the chemical process is divided into a distribution network and process operator. The resulting infinite linear program allows the identification of a global lower bound (Wilson and Manousiouthakis, 2000). Recently, Ryu and Maravelias (2019) proposed a MILP model that uses a discrete temperature grid for the process synthesis problem. In addition, they showed how nonuniform grids can reduce the complexity of large scale problems.

In contrast, the formulation of the FluxMax approach is more general: the discretization of the entire thermodynamic state space and the introduction of a generalized process extent variable allow the direct application of the approach to any kind of synthesis problem. In addition, the introduction of inequalities enables the simultaneous consideration of heat integration as integrated part of the flux optimization problem, so that the prior screening of feasibility is waived, which ensures the consideration of both the temperature levels and the heat fluxes actually transferred. In the following, the digraph concept and the model formulation of the FluxMax approach are presented, which can be regarded as a generalization of earlier work, e.g. (Schack et al., 2018, Liesche et al., 2019). Subsequently the novel heat integration model is introduced, which enables direct and indirect heat integration.

\subsection{Directed graph representation of chemical process networks}

The decoupling of nonlinear preprocessing and subsequent flux optimization is achieved by representing the chemical process network as directed graph (digraph) that consists of nodes and edges. Also Friedler et al. (1992) used a graph representation and introduced material and operating nodes (Cabezas et al., 2018). In contrast, the FluxMax approach distinguishes between four types of nodes (or vertices). Firstly, there are thermodynamic substance nodes $(\mathrm{TSN}) \mathrm{M}_{i} \in \mathcal{M}$ where $\mathcal{M}$ is the set of all TSNs. TSN represent discrete 
state points in the thermodynamic state space. Secondly, there is the set of elementary process nodes $(\mathrm{EPN}) \mathcal{E}$ containing all elementary process nodes $\mathrm{E}_{j} \in \mathcal{E}$ at which any kind of chemical interaction among TSNs takes place. The third group of nodes are utility nodes $(\mathrm{UN}) \mathrm{U}_{l} \in \mathcal{U}$ to provide heating and cooling. Herein, $\mathcal{U}$ is the set of possible utilities at different temperatures. A fourth group of nodes - the work utility nodes (WUN) $\mathrm{S}_{k}$ - are contained in the set $\mathcal{S}$. The WUNs represent the electrical grid that supplies the required electrical power or distribute the generated power, in case of power generating processes.

The nodes are connected by edges, that represent the mass- and energy fluxes desired to be optimized. The set of all fluxes is denoted as $\mathcal{F}$.

\subsubsection{Thermodynamic substance nodes}

Thermodynamic substance nodes (TSN) are discrete points in the thermodynamic state space as introduced in (Liesche et al., 2018). As a consequence, a thermodynamic substance node $\mathrm{M}_{i}$ is clearly defined by its thermodynamic coordinates $\zeta_{z}$, where $z$ corresponds to a dimension of the thermodynamic state space. Examples of thermodynamic coordinates are molar composition $\left[x_{1}, x_{2}, \ldots, x_{i}\right]^{\top}$, temperature $T$, and pressure $p$. For each temperature, pressure or composition change a new TSN is introduced. The thermodynamic potentials, such as enthalpy or entropy, are calculated a priori by using convenient nonlinear equations of state.

In order to move from one $\mathrm{TSN}_{i}$ to another $\mathrm{TSN} \mathrm{M}_{i+1}$, elementary process functions (EPF) are required. In Fig. 2 (A) four different elementary process functions are illustrated for the transformation of TSNs: isobaric isothermal separation $\left(\mathrm{EPF}_{1}\right)$, isobaric isothermal reaction $\left(\mathrm{EPF}_{2}\right)$, isothermal compression $\left(\mathrm{EPF}_{3}\right)$, and isobaric heating $\left(\mathrm{EPF}_{4}\right)$.

\subsubsection{Elementary process node}

Elementary process nodes $\mathrm{E}_{j}$ are introduced to describe the elementary process functions that enable the chemical transformation among TSNs. The mass fluxes, which are illustrated as black arrows in Fig. 2 (B), connect an elementary process node with at least two TSNs. Stoichiometric equations are formulated to describe the transformation between TSNs analogous to pure substances $\alpha \in \mathcal{A}$. Hereby, $\mathcal{A} \subset \mathcal{M}$ is the set of pure substances, 


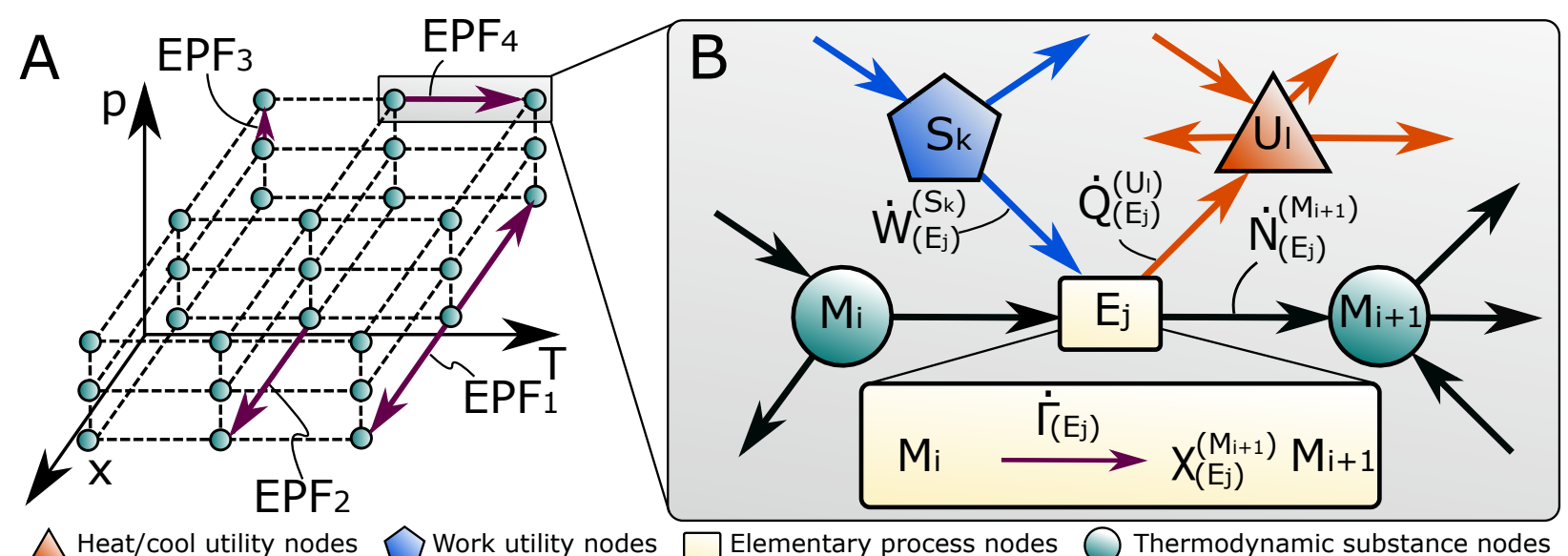

Figure 2: Grid of thermodynamic state points (A) in the thermodynamic state space with $\mathrm{p}, \mathrm{T}$ and $\mathbf{x}$ coordinates. Thermodynamic substance nodes (green) are linked via elementary process functions (magenta): isobaric isothermal separation $\left(\mathrm{EPF}_{1}\right)$; isobaric isothermal reaction $\left(\mathrm{EPF}_{2}\right)$; isothermal compression $\left(\mathrm{EPF}_{3}\right)$; and isobaric heating $\left(\mathrm{EPF}_{4}\right)$. The digraph representation for EPFs that link two TSN (green circles) $\mathrm{M}_{i}$ and $\mathrm{M}_{i+1}$ via an elementary process node $\mathrm{E}_{j}$ (yellow rectangle) is illustrated on the right (B). Work utility nodes (blue pentagon) and heat utility nodes (red triangle) supply the EPN with duties. Work fuxes (blue arrows), heat fuxes (red arrows) and molar fuxes (black arrows) link the four node types. The conversion is described by a stoichiometric equation that is characterized by the generalized process extent number $\dot{\Gamma}_{\left(\mathrm{E}_{j}\right)}$.

which are a special case of TSN. As shown in Fig. 2 (B) the generalized stoichiometric coefficients are denoted as $\chi_{\left(\mathrm{E}_{j}\right)}^{\left(\mathrm{M}_{i}\right)}$. Similarly to the extent of reaction $\dot{\xi}$ that is frequently used for the description of a chemical reactor, a generalized process extent number (PEN) $\dot{\Gamma}$ is introduced that links all participating TSN of an elementary process node. It can be interpreted as an extent of the elementary process:

$$
\mathrm{d} \dot{\Gamma}_{\left(\mathrm{E}_{j}\right)}:=\chi_{\left(\mathrm{E}_{j}\right)}^{\left(\mathrm{M}_{i}\right)} \mathrm{d} \dot{N}_{\left(\mathrm{E}_{j}\right)}^{\left(\mathrm{M}_{i}\right)}
$$

From Eq. (1) follows that $\dot{\Gamma}_{\left(\mathrm{E}_{j}\right)}=0$ if this transformation along $\mathrm{E}_{j}$ is inactive. Contrary to the extent of the reaction that directly affects outlet composition of a reactor flux, the PENs can be considered as a scaling variable that allows an elegant formulation of the flow problem by relating each flow of an EPN to a unique PEN.

In addition, heat and work fluxes, illustrated as blue and red arrows, connect EPNs with utility nodes. 


\subsubsection{Utility nodes}

Utility nodes $\mathrm{U}_{l}$ are introduced to provide the heating and cooling demands of the EPNs. UNs are considered as reservoirs of heat at a constant temperature level. The external heating and cooling requirements are fulfilled by at least two UNs at sufficiently low and high temperature, respectively. In case of indirect heat integration, which is introduced in section 2.3 , the UNs are additionally used to enable the heat integration.

The work utility nodes $\mathrm{S}_{k}$ provide the external power demand.

\subsubsection{Edges representing mass- and energy fluxes}

In a directed graph the nodes are connected with edges. These edges can be weighted or limited in a subsequent optimization problem. In the proposed approach, there are two types of edges. Firstly, there are edges corresponding to mass fluxes that connect at least two TSNs with an elementary process node. As shown in Fig. 2 (B) these fluxes are either external or internal mass fluxes (black arrows). In case of external fluxes, the fluxes are a consequence of initially provided substances, which are desired to be transformed within the chemical process, or the final products, which leave the overall process. In contrast, internal mass fluxes are fluxes among elementary processes and thus in- and outlet flows of the EPNs.

Secondly, there are heat and work fluxes that connect the UNs and WUNs with EPNs as illustrated as red and blue arrows in Fig. 2 (B).

\subsection{Formulation of node conservation laws}

In this section, the conservation laws of the introduced nodes are presented, that are used as equality constraints in the subsequently formulated flux optimization problem. For the thermodynamic substance nodes only mass balances are formulated, because the thermodynamic state of a TSN is clearly determined by its thermodynamic coordinates. For utility nodes only energy balances are formulated as they are not connected to mass fluxes. In contrast, for the elementary process both mass and energy balances have to be formulated. It should be mentioned, that in this study the work demand of $\mathrm{E}_{j}$ is considered as external work supply. As a consequence, the energy balance for WUNs is omitted. 
Once the elementary processes are described with stoichiometric equations that link TSNs, mass and energy balances for each elementary process node $\mathrm{E}_{j}$ are formulated. In contrast to the formulation of partial mass balances for each substance in classical modeling approaches, mass balances for TSN are formulated directly by making use of the PENs $\dot{\Gamma}$ : all balances have a similar format because they link one TSN $\mathrm{M}_{i}$ with the PEN for each elementary process $\mathrm{E}_{j}$ via their stoichiometric coefficients $\chi_{\left(\mathrm{E}_{j}\right)}^{\left(\mathrm{M}_{i}\right)}$ :

$$
0=-\operatorname{sgn}\left(\chi_{\left(\mathrm{E}_{j}\right)}^{\left(\mathrm{M}_{i}\right)}\right) \dot{N}_{\left(\mathrm{E}_{j}\right)}^{\left(\mathrm{M}_{i}\right)}+\chi_{\left(\mathrm{E}_{j}\right)}^{\left(\mathrm{M}_{i}\right)} \dot{\Gamma}_{\left(\mathrm{E}_{j}\right)} \quad \forall \mathrm{E}_{j} \in \mathcal{E} ; \forall \mathrm{M}_{i} \in \mathcal{M}
$$

The energy demands of $\mathrm{E}_{j}$ are expressed by the specific, molar heat $(\varphi)$ and work $(\omega)$ duties, which are calculated a priori by suitable (nonlinear) models. The generic system of three equations that constitutes the total energy balance for unit $\mathrm{E}_{j}$ is formulated as:

$$
\begin{aligned}
0= & \left(-\omega_{\left(\mathrm{E}_{j}\right)}^{\text {in }}+\omega_{\left(\mathrm{E}_{j}\right)}^{\text {out }}\right) \dot{\Gamma}_{\left(\mathrm{E}_{j}\right)}+\dot{W}_{\left(\mathrm{E}_{j}\right)}^{\text {ext, in }}-\dot{W}_{\left(\mathrm{E}_{j}\right)}^{\text {ext out }} \\
0= & {\left[\varphi_{\left(\mathrm{E}_{j}\right)}^{\text {out }}+\left(1-\eta_{\left(\mathrm{E}_{j}\right)}^{\text {in }}\right) \omega_{\left(\mathrm{E}_{j}\right)}^{\text {in }}+\left(\frac{1}{\eta_{\left(\mathrm{E}_{j}\right)}^{\text {out }}}-1\right) \omega_{\left(\mathrm{E}_{j}\right)}^{\text {out }}\right] \dot{\Gamma}_{\left(\mathrm{E}_{j}\right)}-\sum_{\mathrm{U}_{l} \in \mathcal{U}} \dot{Q}_{\left(\mathrm{U}_{l}\right)}^{\left(\mathrm{E}_{j}\right)} } \\
0= & -\varphi_{\left(\mathrm{E}_{j}\right)}^{\text {in }} \dot{\Gamma}_{\left(\mathrm{E}_{j}\right)}+\sum_{\mathrm{U}_{l} \in \mathcal{U}} \dot{Q}_{\left(\mathrm{E}_{j}\right)}^{\left(\mathrm{U}_{l}\right)} \\
& \forall \mathrm{E}_{j} \in \mathcal{E}
\end{aligned}
$$

where $\dot{Q}_{\left(\mathrm{U}_{l}\right)}^{\left(\mathrm{E}_{j}\right)}, \dot{Q}_{\left(\mathrm{E}_{j}\right)}^{\left(\mathrm{U}_{l}\right)}, \dot{W}_{\left(\mathrm{E}_{j}\right)}^{\text {ext out }}, \dot{W}_{\left(\mathrm{E}_{j}\right)}^{\text {ext, in }} \in \mathbb{R}_{0}^{+}$. The superscript of an internal flow $\dot{Q}_{\left(\mathrm{E}_{j}\right)}^{\left(\mathrm{U}_{l}\right)}$ or $\dot{Q}_{\left(\mathrm{U}_{l}\right)}^{\left(\mathrm{E}_{j}\right)}$, respectively, indicates the node from which it originates and the subscript its destination node. It is important to note, that these heat fluxes correspond directly to the external heating and cooling supply if no heat integration is considered: $\sum \dot{Q}_{\left(\mathrm{E}_{j}\right)}^{\left(\mathrm{U}_{l}\right)}=\dot{Q}_{\left(\mathrm{E}_{j}\right)}^{\text {ext, in }}$ and $\sum \dot{Q}_{\left(\mathrm{U}_{l}\right)}^{\left(\mathrm{E}_{j}\right)}=\dot{Q}_{\left(\mathrm{E}_{j}\right)}^{\mathrm{ext} \text { out }}$

The reason to split the overall energy balance into three balances is that in this way the consideration of a dedicated entropy balances is avoided: Eq. (3a) determines the external 
work fluxes $\dot{W}_{\left(\mathrm{E}_{j}\right)}^{\text {ext, in }}$ and $\dot{W}_{\left(\mathrm{E}_{j}\right)}^{\text {ext, out }}$, which depend on the molar work demand $\omega_{\left(\mathrm{E}_{j}\right)}^{\text {in }}$ or generation $\omega_{\left(\mathrm{E}_{j}\right)}^{\text {out }}$ of $\mathrm{E}_{j}$. Here, the assumption was made that simultaneous work in- and output is not allowed, which is fulfilled for the case study under consideration. However, if also work generating EPNs are considered, the balances are easily adjustable. Eq. (3b) determines the cooling requirement of $\mathrm{E}_{j}$, that consists of three contributions: cooling duty $\varphi_{\left(\mathrm{E}_{j}\right)}^{\text {out }}$ e.g. due to condensation and cooling duties resulting from waste heat for work in- and output flows which is accounted for by means of two efficiency factors $\eta_{\left(\mathrm{E}_{j}\right)}^{\text {in }}$ and $\eta_{\left(\mathrm{E}_{j}\right)}^{\text {out }}$ :

$$
\eta_{\left(\mathrm{E}_{j}\right)}^{\text {in }}:=\frac{\omega_{\left(\mathrm{E}_{j}\right)}^{\text {in, rev }}}{\omega_{\left(\mathrm{E}_{j}\right)}^{\text {in }}} \quad \text { and } \quad \eta_{\left(\mathrm{E}_{j}\right)}^{\text {out }}:=\frac{\omega_{\left(\mathrm{E}_{j}\right)}^{\text {out }}}{\omega_{\left(\mathrm{E}_{j}\right)}^{\text {out, rev }}}
$$

The third Eq. (3c) determines the heating demand $\dot{Q}_{\left(\mathrm{E}_{j}\right)}^{\left(\mathrm{U}_{l}\right)}$ of $\mathrm{E}_{j}$, which depends on the molar heat duty $\varphi_{\left(\mathrm{E}_{j}\right)}^{\text {in }}$.

Selected examples of elementary processes and corresponding mass- and energy balances are listed in the supplementary material in Tab. S1.

After introducing balances for the EPNs, the mass balances for thermodynamic state nodes are formulated as:

$$
0=\sum_{\mathrm{E}_{j} \in \mathcal{E}} \operatorname{sgn}\left(\chi_{\left(\mathrm{E}_{j}\right)}^{\left(\mathrm{M}_{i}\right)}\right) \dot{N}_{\left(\mathrm{E}_{j}\right)}^{\left(\mathrm{M}_{i}\right)}+\dot{N}_{\mathrm{ext}, \text { in }}^{\left(\mathrm{M}_{i}\right)}-\dot{N}_{\mathrm{ext}, \text { out }}^{\left(\mathrm{M}_{i}\right)} \quad \forall \mathrm{M}_{i} \in \mathcal{M}
$$

taking into account all internal mass fluxes $\dot{N}_{\left(\mathrm{E}_{j}\right)}^{\left(\mathrm{M}_{i}\right)}$ that link $\mathrm{E}_{j}$ and $\mathrm{M}_{i}$ as well as external mass fluxes that provide the initial reactants $\dot{N}_{\text {ext, in }}^{\left(\mathrm{M}_{i}\right)}$ or release the final products $\dot{N}_{\text {ext, out }}^{\left(\mathrm{M}_{i}\right)}$. The sign of the stoichiometric coefficient $\chi_{\left(\mathrm{E}_{j}\right)}^{\left(\mathrm{M}_{i}\right)}$ denotes the direction of the internal mass flux between $\mathrm{E}_{j}$ and $\mathrm{M}_{i}$. No energy balances are required for TSNs, as the thermodynamic state of all fluxes, that are connected with a TSN, is equal by definition. As a consequence, two TSNs are not linked directly.

In contrast, for each utility node $\mathrm{U}_{l}$ an energy balance has to be formulated, which 
simplifies to a single heat balance:

$$
0=\sum_{\mathrm{E}_{j} \in \mathcal{E}}\left(\dot{Q}_{\left(\mathrm{U}_{l}\right)}^{\left(\mathrm{E}_{j}\right)}-\dot{Q}_{\left(\mathrm{E}_{j}\right)}^{\left(\mathrm{U}_{l}\right)}\right)+\dot{Q}_{\left(\mathrm{U}_{l}\right)}^{\text {ext,in }}-\dot{Q}_{\left(\mathrm{U}_{l}\right)}^{\text {ext,out }} \quad \forall \mathrm{U}_{l} \in \mathcal{U}
$$

The sum of all heat fluxes that enter a utility $\mathrm{U}_{l}$ - heat fluxes $\dot{Q}_{\left(\mathrm{U}_{l}\right)}^{\left(\mathrm{E}_{i}^{\text {out }}\right)}$ from $\mathrm{E}_{j}$ and externally provided heat fluxes $\dot{Q}_{\left(\mathrm{U}_{l}\right)}^{\text {ext,in }}$ - have to be equal to all heat fluxes that leave a utility $\mathrm{U}_{l}$ - heat fluxes $\dot{Q}_{\left(\mathrm{E}_{j}^{\mathrm{in}}\right)}^{\left(\mathrm{U}_{l}\right)}$ to $\mathrm{E}_{j}$ and externally released heat fluxes $\dot{Q}_{\left(\mathrm{U}_{l}\right)}^{\text {ext,out }}$.

\subsection{Heat integration model}

Pinch analysis is widely used to invent optimal heat exchanger networks for a given process structure, which consists of hot and cold heat streams (Linnhoff and Flower, 1978). In this context, hot streams are defined as streams to be cooled and cold streams to be heated. However, Pinch analysis cannot be applied directly to a simultaneous optimization approach, as the heat duties are decision variables and thus not known a priori. As a consequence, often a subsequent procedure is proposed in literature: first the flow problem is optimized without consideration of heat integration and subsequently a Pinch-based analysis is performed to identify the heat integration potential (Kokossis et al., 2015, Ulonska et al., 2016, Gençer and Agrawal, 2018). These approaches may not identify the overall massand energetic optimum (Duran and Grossmann, 1986). To identify the global mass- and energetic optimum, a simultaneous procedure is crucial that optimize simultaneously the flow problem under consideration of heat integration.

While in other approaches the feasibility of heat integration need to be ensured a priori (Holiastos and Manousiouthakis, 2004, Pichardo and Manousiouthakis, 2017), the FluxMax approach ensures the feasibility by introducing inequality constraints that enables the simultaneous heat integration. Only in this way is the internally transferred heat flux limited, which ensures an adequate calculation of the actual heat integration potential. The presented constraints allow direct - among entities - as well as indirect - via utilities - heat integration.

While for direct heat integration, the heat is directly transferred among entities (Fig. $3(\mathrm{~A})$ ), 

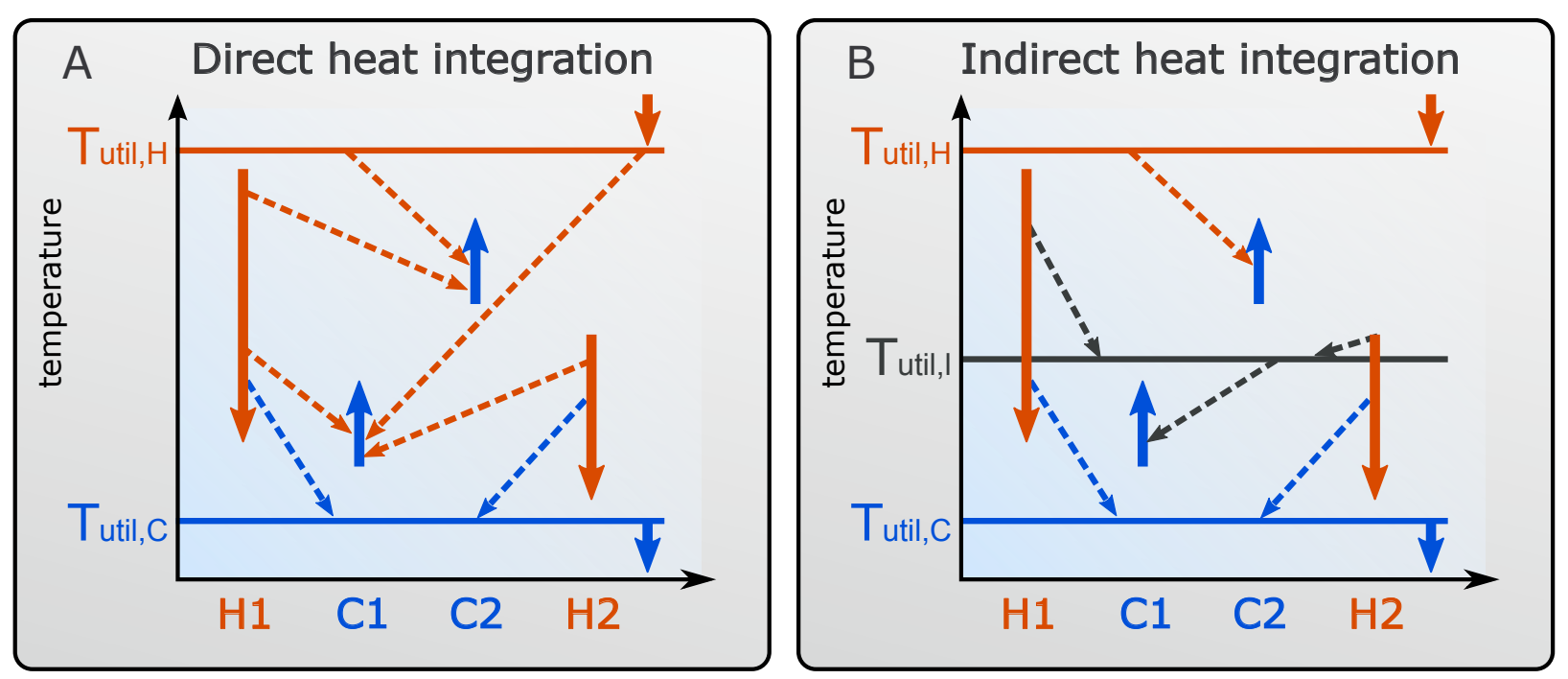

Figure 3: Schematic illustration of direct and indirect heat integration; A) direct heat integration: hot $(\mathrm{H})$ and cold streams (C) interact directly, utilities are only used to provide external heating and cooling at sufficiently high $T_{\mathrm{util}, \mathrm{H}}$ and low temperature $\left.T_{\mathrm{util}, \mathrm{C}} ; \mathrm{B}\right)$ indirect heat integration: introduction of additional utilities at intermediate temperatures $T_{\mathrm{util}, 1}$ that facilitate heat integration.

indirect heat integration utilizes the utility nodes to enable heat integration (Fig. 3 (B)). The maximum amount of internally transferable heat depends on the temperature levels of cold and hot streams. Analogous to Pinch analysis, cold streams require heat whereas hot streams provide heat. Three distinct temperature domains can be distinguished, that determine the heat integration possibility depending on the minimum temperature difference $\Delta T_{\min }$ and the in- and outlet temperatures of hot streams $T_{\mathrm{H}, \text { in }}$ and $T_{\mathrm{H}, \text { out }}$, or of cold streams $T_{\mathrm{C}, \text { in }}$ and $T_{\mathrm{C} \text {,out }}$, respectively: domain I: total heat integration possible, domain II: partial heat integration possible, and domain III: heat integration infeasible. The temperature condition and classification of hot and cold fluxes are listed in Table 1.

Table 1 shows that a cold stream can be completely heated internally by hot streams (domain I) if the maximum inlet temperature $T_{\mathrm{H}, \mathrm{in}}^{\max }$ of the corresponding hot streams is higher than the cold outlet stream temperature and a minimum temperature difference, that ensures a sufficiently large driving force. In contrast, heat integration is infeasible if the cold inlet stream temperature is larger than the hot inlet stream temperature. In the other cases (domain II), the cold stream can be partially heated internally. An analogous classification can be done for hot streams that have to be cooled internally by cold streams 
Table 1: Temperature Conditions for for Classification of Heat Integration Possibility.

\begin{tabular}{|c|c|c|}
\hline Domain & Condition & Heat Integration \\
\hline \multicolumn{3}{|c|}{ Cold Fluxes } \\
\hline $\mathrm{I}$ & i) $T_{\mathrm{H}, \text { in }}^{\max } \geq T_{\mathrm{C}, \text { out }}+\Delta T_{\min }$ & total \\
\hline II & $\begin{array}{l}\text { i) } T_{\mathrm{H}, \text { in }}^{\max }<T_{\mathrm{C}, \text { out }}+\Delta T_{\min } \\
\text { ii) } T_{\mathrm{H}, \text { in }}^{\max }>T_{\mathrm{C}, \text { in }}+\Delta T_{\min }\end{array}$ & partial \\
\hline III & i) $T_{\mathrm{H}, \text { in }}^{\max } \leq T_{\mathrm{C}, \text { in }}+\Delta T_{\min }$ & infeasible \\
\hline \multicolumn{3}{|c|}{ Hot Fluxes } \\
\hline I & i) $T_{\mathrm{C}, \text { in }}^{\min }+\Delta T_{\min } \leq T_{\mathrm{H}, \text { out }}$ & total \\
\hline II & $\begin{array}{l}\text { i) } T_{\mathrm{C}, \text { in }}^{\min }+\Delta T_{\min }<T_{\mathrm{H}, \text { in }} \\
\text { ii) } T_{\mathrm{C}, \text { in }}^{\min }+\Delta T_{\min }>T_{\mathrm{H}, \text { out }}\end{array}$ & partial \\
\hline III & i) $T_{\mathrm{C}, \text { in }}^{\min }+\Delta T_{\min } \geq T_{\mathrm{H}, \text { in }}$ & infeasible \\
\hline
\end{tabular}

depending on the minimum inlet temperature of cold streams $T_{\mathrm{C}, \text { in }}^{\min }$ (Table 1 ).

The internal heat fluxes that supply heat to a cold EPN $\mathrm{E}_{j}$ are denoted as $\dot{Q}_{\left(\mathrm{E}_{j}\right)}^{\left(k_{j}^{H}\right)}$, while $\dot{Q}_{\left(k_{m j}^{C}\right)}^{\left(\mathrm{E}_{j}\right)}$ originate from a hot $\mathrm{EPN} \mathrm{E}_{j}$. Hereby, $k_{m j}^{H} \in \mathcal{E}, k_{m j}^{C} \in \mathcal{E}$ for direct heat integration and $k_{m j}^{H} \in \mathcal{U}, k_{m j}^{C} \in \mathcal{U}$ for indirect heat integration. For stream combinations that belong to domain I, no inequality has to be formulated, because the heating or cooling demand of the corresponding EPN can be completely provided internally. However, for combinations that belong to domain II the maximum amount of transferable heat has to be quantified. Two subsets of $\mathcal{F}$ are introduced: i) the subset $\mathcal{F}_{\mathrm{II}}^{H, \mathrm{E}_{j}}:=\left\{\dot{Q}_{\left(\mathrm{E}_{j}\right)}^{\left(k_{m j}^{H}\right)} \in \mathbb{R}_{0}^{+} \mid\right.$case II satisfied $\}$that contains all the internal heat fluxes that supply heat to a cold EPN $\mathrm{E}_{j}$; and ii) the subset $\mathcal{F}_{\mathrm{II}}^{\mathrm{E}_{j}, C}:=\left\{\dot{Q}_{\left(k_{m j}^{C}\right)}^{\left.\left(\mathrm{E}_{j}\right)\right)} \in \mathbb{R}_{0}^{+} \mid\right.$case II satisfied $\}$that contains all the internal heat fluxes that release heat from a hot $\mathrm{EPN}_{j}$. The first subset $\mathcal{F}_{\mathrm{II}}^{h, \mathrm{E}_{j}}$ is used to formulate an inequality for every combination of cold EPN $\mathrm{E}_{j}^{\text {in }}$ and possibly interacting hot streams that belong to domain II:

$$
0 \leq \frac{T_{\mathrm{H}, \text { in }}^{\max }-T_{\mathrm{C}, \mathrm{in},\left(\mathrm{E}_{\mathrm{j}}\right)}-\Delta T_{\mathrm{min}}}{T_{\mathrm{C}, \text { out },\left(\mathrm{E}_{\mathrm{j}}\right)}-T_{\mathrm{C}, \mathrm{in},\left(\mathrm{E}_{\mathrm{j}}\right)}} \varphi_{\left(\mathrm{E}_{\mathrm{j}}\right)}^{\mathrm{in}} \dot{\Gamma}_{\left(\mathrm{E}_{\mathrm{j}}\right)}-\sum_{k_{m j}^{H} \in \mathcal{K}_{m}^{H}} \dot{Q}_{\left(\mathrm{E}_{j}\right)}^{\left(k_{m j}^{H}\right)} \quad \forall \dot{Q}_{\left(\mathrm{E}_{j}\right)}^{\left(k_{m j}^{H}\right)} \in \mathcal{K}_{m}^{H}
$$

Herein, $\varphi_{\left(\mathrm{E}_{j}\right)}^{\mathrm{in}}$ denotes the specific heat demand of $\mathrm{E}_{j}^{\mathrm{in}}$ and $T_{\mathrm{Hot}, \mathrm{in}}^{\mathrm{max}}$ the maximum inlet temperature of the possibly interacting hot streams. $\mathcal{K}_{m}^{H} \subset \mathcal{K}^{H}=\left\{k_{m j}^{H}\right\}$ denotes the $m$-th row of the set of all permutations of $\mathcal{F}_{\mathrm{II}}^{H, \mathrm{E}_{j}}$, that determine all possibly interacting streams of 
cold EPN $\mathrm{E}_{j}$. Illustrated for an example of three possible interacting hot streams $\left(\mathrm{H}_{1}, \mathrm{H}_{2}, \mathrm{H}_{3}\right)$ the set of permutations $\mathcal{K}^{H}$ equals to:

$$
\mathcal{K}^{H}=\left\{\begin{array}{lll}
\mathrm{H}_{1} & & \\
& \mathrm{H}_{2} & \\
& & \mathrm{H}_{3} \\
\mathrm{H}_{1} & \mathrm{H}_{2} & \\
\mathrm{H}_{1} & & \mathrm{H}_{3} \\
& \mathrm{H}_{2} & \mathrm{H}_{3} \\
\mathrm{H}_{1} & \mathrm{H}_{2} & \mathrm{H}_{3}
\end{array}\right\}
$$

In the same way, an inequality for every combination of hot EPN $\mathrm{E}_{j}$ and possibly interacting cold streams is introduced by using the specific excess of heat $\varphi_{\left(\mathrm{E}_{\mathrm{j}}\right)}^{\text {out }}$, the minimum inlet temperature of possibly interacting cold streams $T_{\mathrm{C}, \text { in }}^{\min }$, the subset $\mathcal{F}_{\mathrm{II}}^{\mathrm{E}_{j}, C}$, and the corresponding set of permutations $\mathcal{K}^{C}$, according to Eq. (8):

$$
0 \leq \frac{T_{\mathrm{H}, \mathrm{in},\left(\mathrm{E}_{j}\right)}-T_{\mathrm{C}, \mathrm{in}}^{\mathrm{min}}-\Delta T_{\mathrm{min}}}{T_{\mathrm{H}, \mathrm{in},\left(\mathrm{E}_{j}\right)}-T_{\mathrm{H}, \mathrm{out},\left(\mathrm{E}_{j}\right)}^{\text {out }}} \dot{\Gamma}_{\left(\mathrm{E}_{j}\right)} \dot{\Gamma}_{\left(\mathrm{E}_{j}\right)}-\sum_{k_{m j}^{C} \in \mathcal{K}_{m}^{C}} \dot{Q}_{\left(\mathrm{k}_{\mathrm{mj}}^{\mathrm{C}}\right)}^{\left(\mathrm{E}_{j}\right)} \quad \forall \dot{Q}_{\left(\mathrm{k}_{\mathrm{mj}}^{\mathrm{C}}\right)}^{\left(\mathrm{E}_{j}\right)} \in \mathcal{K}_{m}^{C}
$$

The maximum inlet temperature of possibly interacting hot streams $T_{\mathrm{H} \text {,in }}^{\max }$ and the minimum inlet temperature of possibly interacting cold streams $T_{\mathrm{C}, \text { in }}^{\mathrm{min}}$ are calculated by comparing the temperatures of the interacting partner streams as shown in Eqs. (10a) and (10b).

$$
\begin{aligned}
T_{\mathrm{H}, \text { in }}^{\max } & =\max _{k \in \mathcal{K}_{H}}\left(T_{k, \text { in }}\right) \\
T_{\mathrm{C}, \mathrm{in}}^{\min } & =\min _{k \in \mathcal{K}_{C}}\left(T_{k, \text { in }}\right)
\end{aligned}
$$

After introducing the general heat integration model, in the following two different approaches - direct and indirect heat integration - are presented, which mainly differ in the considered sets of permutations $\mathcal{K}_{H}$ and $\mathcal{K}_{C}$ of possibly interacting hot or cold streams, respectively. 


\subsubsection{Direct heat integration among elementary process nodes}

In case of direct heat integration, the UNs provide the external heating and cooling at sufficient high and low temperature as shown in Fig. 3 (A). Thus, according to the classification in three domains the heat fluxes that link UNs and EPNs belong to domain I. To enable internal heat transfer, additional heat flux variables $\dot{Q}_{\left(\mathrm{E}_{i}\right)}^{\left(\mathrm{E}_{j}\right)}$ and $\dot{Q}_{\left(\mathrm{E}_{j}\right)}^{\left(\mathrm{E}_{i}\right)}$, that link two EPNs directly, have to be added in the energy balances:

$$
\begin{aligned}
0= & \left(-\omega_{\left(\mathrm{E}_{j}\right)}^{\text {in }}+\omega_{\left(\mathrm{E}_{j}\right)}^{\text {out }}\right) \dot{\Gamma}_{\left(\mathrm{E}_{j}\right)}+\dot{W}_{\left(\mathrm{E}_{j}\right)}^{\text {ext, in }}-\dot{W}_{\left(\mathrm{E}_{j}\right)}^{\text {ext, out }} \\
0= & {\left[\varphi_{\left(\mathrm{E}_{j}\right)}^{\text {out }}+\left(1-\eta_{\left(\mathrm{E}_{j}\right)}^{\text {in }}\right) \omega_{\left(\mathrm{E}_{j}\right)}^{\text {in }}+\left(\frac{1}{\eta_{\left(\mathrm{E}_{j}\right)}^{\text {out }}}-1\right) \omega_{\left(\mathrm{E}_{j}\right)}^{\text {out }}\right] \dot{\Gamma}_{\left(\mathrm{E}_{j}\right)}-\sum_{\mathrm{U}_{l} \in \mathcal{U}} \dot{Q}_{\left(\mathrm{U}_{l}\right)}^{\left(\mathrm{E}_{j}\right)}-\sum_{\mathrm{E}_{i} \in \mathcal{E}} \dot{Q}_{\left(\mathrm{E}_{i}\right)}^{\left(\mathrm{E}_{j}\right)} } \\
0= & -\varphi_{\left(\mathrm{E}_{j}\right)}^{\text {in }} \dot{\Gamma}_{\left(\mathrm{E}_{j}\right)}+\sum_{\mathrm{U}_{l} \in \mathcal{U}} \dot{Q}_{\left(\mathrm{E}_{j}\right)}^{\left(\mathrm{U}_{l}\right)}+\sum_{\mathrm{E}_{i} \in \mathcal{E}} \dot{Q}_{\left(\mathrm{E}_{j}\right)}^{\left(\mathrm{E}_{i}\right)} \\
& \forall \mathrm{E}_{j} \in \mathcal{E}
\end{aligned}
$$

whereby according to Fig. 3 (A), the assumption of only two utility nodes - one at sufficient high, and one at sufficient low temperature - can be made, which result in:

$$
\begin{aligned}
& \sum_{\mathrm{U}_{l} \in \mathcal{U}} \dot{Q}_{\left(\mathrm{E}_{j}\right)}^{\left(\mathrm{U}_{l}\right)}=\dot{Q}_{\left(\mathrm{E}_{j}\right)}^{\mathrm{ext}, \mathrm{in}} \\
& \sum_{\mathrm{U}_{l} \in \mathcal{U}} \dot{Q}_{\left(\mathrm{U}_{l}\right)}^{\left(\mathrm{E}_{j}\right)}=\dot{Q}_{\left(\mathrm{E}_{j}\right)}^{\mathrm{ext}, \text { out }}
\end{aligned}
$$

Adding the newly introduced internal heat flux variables in every energy balance, as shown in Eq. (11), and the introduction of inequalities (Eqs. (7) and (9)), facilitates the simultaneous consideration of heat integration as a part of the optimization problem. However, the number of inequalities increase drastically due to the increasing combinatorial complexity if the number of entities increases. Therefore, in the next section indirect heat integration via utilities is presented. 


\subsubsection{Indirect heat integration via utility nodes}

In chemical production plants, heat is usually not transferred directly among individual process units, but via a network of utilities. Utilities are considered as reservoirs of heat at a constant temperature, such as steam at a specific pressure, or a sufficiently large water reservoir (e.g. a river). Depending on the temperature levels of heat demanding or heat supplying EPN, respectively, the utilities serve either as heat source or sink, as shown in Fig. 3.

In contrast to direct heat integration, further utility nodes at intermediate temperatures are considered as shown in Fig. $3(\mathrm{~B})$. As a consequence, the heat fluxes $\dot{Q}_{\left(\mathrm{E}_{j}\right)}^{\left(\mathrm{U}_{l}\right)}$ and $\dot{Q}_{\left(\mathrm{U}_{l}\right)}^{\left(\mathrm{E}_{j}\right)}$ that link UNs and EPNs may belong also to domain II and III. As a consequence, additional inequalities have to be formulated for combinations that belong to domain II, according to Eqs. (7) and (9). However, no additional heat flux variables have to be introduced, as is was the case for direct heat integration. In this way, the complexity of the resulting optimization problem is reduced drastically compared to direct heat integration, since the number of utilities considered is lower than the number of entities, which result in a significant reduction of considered inequalities.

\subsection{Formulation of the optimization problem}

The equalities and inequalities constraints result from the conservation laws and the heat integration conditions. All constraints are linear in terms of the fluxes - mass, heat, and work - which are decision variables of the optimization problem. The general formulation of an optimization problem with linear constraints is shown in Eq. (13).

$$
\begin{aligned}
& \min _{\varphi} f(\boldsymbol{\varphi}) \\
& \text { s.t. } \mathbf{A}_{\text {eq }} \varphi=b_{\text {eq }} \\
& \mathrm{A}_{\mathrm{iq}} \boldsymbol{\varphi} \leq \mathrm{b}_{\mathrm{iq}} \\
& \varphi_{\mathrm{lb}} \leq \varphi \leq \varphi_{\mathrm{ub}}
\end{aligned}
$$

Herein, $f$ is the objective function and $\varphi=(\dot{\mathbf{N}}, \dot{\Gamma}, \dot{\mathbf{Q}}, \dot{\mathbf{W}})^{\top}$ denotes the vector of all decision variables, namely mass fluxes $\dot{\mathbf{N}}$, heat fluxes $\dot{\mathbf{Q}}$, work fluxes $\dot{\mathbf{W}}$, and generalized 
process extent number $\dot{\boldsymbol{\Gamma}}$.

The equality constraints, that are described by the coefficient matrix $\mathbf{A}_{\text {eq }}$ and the vector of right-hand sides $\mathbf{b}_{\text {eq }}$, contain all energy and and mass balances of TSNs, EPNs, and UNs. In addition, $\mathbf{A}_{\mathrm{eq}}$ contains the information of the coupling of the nodes, that result from the generalized utilization of stoichiometric equation.

The temperature constraints, that result from heat integration are summarized by the coefficient matrix of inequalities $\mathbf{A}_{\mathrm{iq}}$ and corresponding right hand sides $\mathbf{b}_{\mathrm{iq}}$. The coefficient matrices, $\mathbf{A}_{\mathrm{eq}}$ and $\mathbf{A}_{\mathrm{iq}}$, and the solution vectors, $\mathbf{b}_{\mathrm{eq}}$ and $\mathbf{b}_{\mathrm{iq}}$, as well as the lower $\boldsymbol{\varphi}_{\mathrm{lb}}$ and upper bounds $\varphi_{\mathrm{ub}}$ vary depending on the chosen application.

\section{Case study}

In recent years methanol $\left(\mathrm{CH}_{3} \mathrm{OH}\right)$ has often been discussed as an important energy storage molecule in Renewables-to-Chemicals (R2Chem) applications (Surya Prakash et al., Bertau et al., 2014). The conversion of carbon dioxide and hydrogen generated by electrolysis is an auspicious way to store so-called electrical surplus energy (Rihko-Struckmann et al., 2010, Moioli et al., 2019). In particular, the low global warming potential was highlighted by König et al. (2019), while the cost are higher compared to bio-based production pathways. Low cost for renewable electricity in the range of approx. $2.5 \mathrm{ct} / \mathrm{kWh}$ would be necessary for an economically competitive production (Ouda et al., 2019). This result is consistent with our previous findings in Schack et al. (2018), where we have also demonstrated the possibility of an economic production of methanol for a combined use of renewable and fossil resources. In addition, simple and well-known production technologies (Ott et al., 2000) and favorable chemical properties of methanol, e.g. $\mathrm{CH}_{3} \mathrm{OH}$ is liquid under ambient conditions, are reasons for the selection of methanol as a target molecule.

\subsection{Methanol synthesis process}

While the focus of previous studies, at which the FluxMax approach is based on, was on the production system level by analyzing the specific methanol production costs and the corresponding $\mathrm{CO}_{2}$ emissions (Schack et al., 2018), or on the process level by optimizing the 
reactor and the compressor cascade design (Liesche et al., 2018), this paper examines the entire methanol production process at the plant level.

The following six elementary processes are considered to model the methanol synthesis process : i) isothermal isobaric reaction, ii) isothermal isobaric separation, iii) isothermal compression, iv) isenthalpic expansion, v) isobaric heating and cooling, and vi) isothermal isobaric mixing. For each elementary process a distinct elementary process node $\mathrm{E}_{j}$ is introduced which is described by a stoichiometric equation introducing stoichiometric coefficients $\chi_{\left(E_{j}\right)}$. In addition, each elementary process is characterized by a specific energy demand for heat $(\varphi)$ and power $(\omega)$.

There are many process technologies to chemically activate $\mathrm{CO}_{2}$ by conversion to carbon monoxide (CO), such as reforming or reverse-water-gas-shift. In Schack et al. (2018) we showed that the preferred technology depends strongly on the energy source. Therefore, the $\mathrm{CO}_{2}$ activation step and corresponding side reactions are neglected in the following, since the focus of this contribution is on the illustration of the FluxMax approach and its key features instead of the overall process analysis. This simplification results in only one reaction equation:

$$
\mathrm{CO}+2 \mathrm{H}_{2} \longrightarrow \mathrm{CH}_{3} \mathrm{OH} \quad \Delta_{R} h^{\ominus}=-90.77 \mathrm{~kJ} / \mathrm{mol} \text {. }
$$

In addition to the technical applied operation conditions, the thermodynamic feasibility has to be taken into account. Fig. 4 shows the pressure dependence of the chemical equilibrium and the boiling temperature of methanol. The feasible reaction conditions are depicted as magenta area. While the maximum amount of methanol in the reactor outlet is determined by the chemical equilibrium (Fig. $4 \mathrm{~A}$ ), the minimum reaction temperature is characterized by the boiling temperature (Fig. 4 B) to ensure that methanol is gaseous.

Due to the simplified reaction system, the reactor outlet flow only consists of condensable methanol and non-condensable, unconverted reactants. As a consequence, the separation can be considered as condensation of methanol. Thus, the separation temperature is set to the boiling temperature of methanol (Fig. 4 B) at the corresponding pressure. 

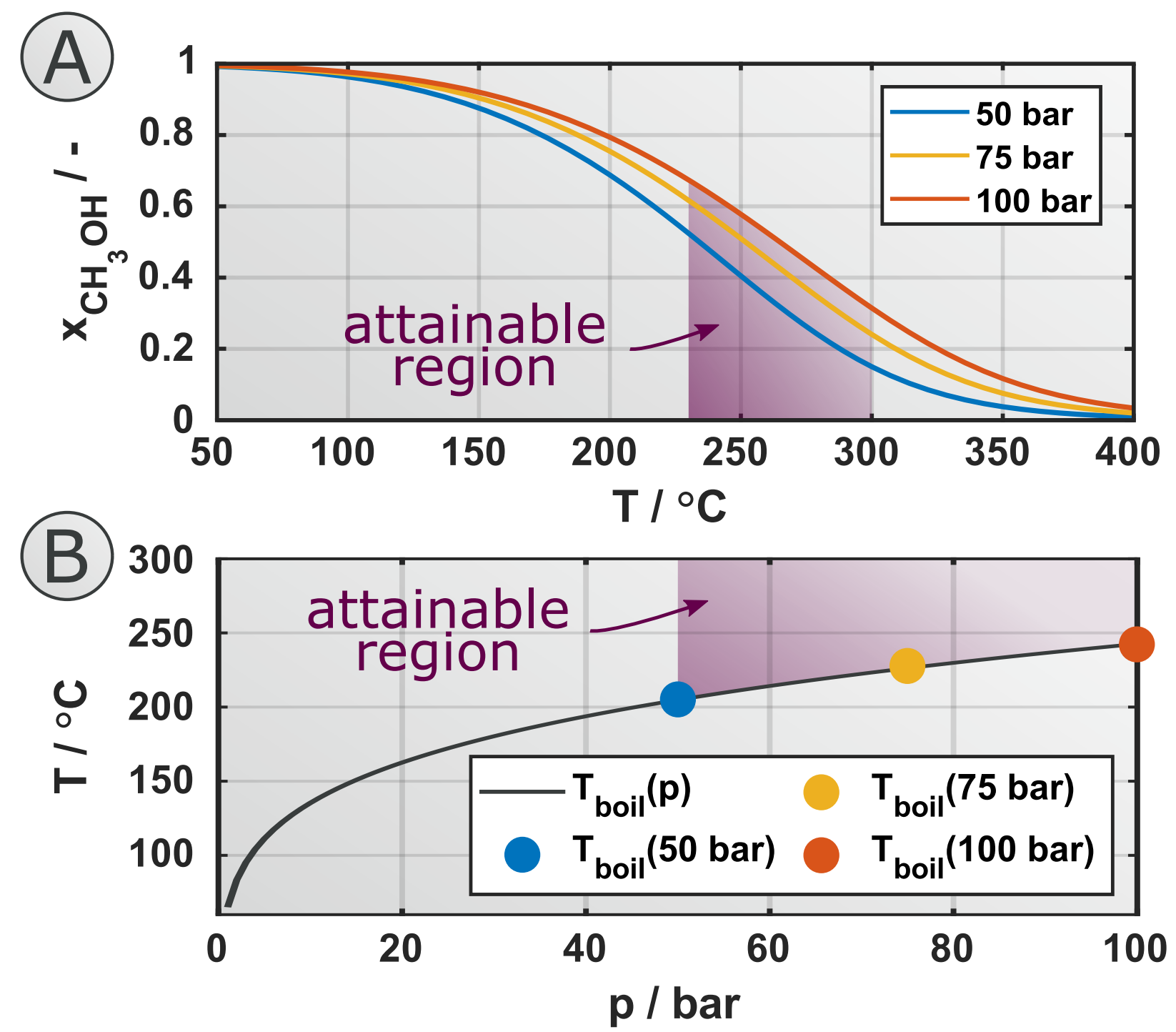

Figure 4: Illustration of the pressure dependence of the chemical equilibrium molar fraction of methanol in the reactor $x_{\mathrm{CH}_{3} \mathrm{OH}}(\mathrm{A})$ and of the boiling temperature of methanol (B); the feasible region for the methanol synthesis reaction is depicted as magenta area. 
The annual production of pure methanol is desired to be $100,000 \mathrm{t}_{\mathrm{CH}_{3} \mathrm{OH}}$ /a. The detailed derivation of the discretization of introduced elementary process can be found in the supplementary material.

\subsection{Objective function}

The conservation laws and temperature conditions for heat integration, introduced in section 2.2 and 2.3, are equality and inequality constraints of the optimization problem. As the constraints are linear in terms of the decision variables, the feasible region is convex. As a consequence, the identification of a global optimum is guaranteed for a convex objective function.

One of the major cost drivers in the field of Renewable-to-Chemicals applications is energy demand. In order to become more competitive compared to fossil-based processes, the energy efficiency of the processes must be increased. This study uses the FluxMax approach to identify energy optimal process configurations. The objective function $f$ is therefore to minimize the total external energy duty - sum of external heating, cooling, and electrical energy - which is linear in terms of the fluxes:

$$
f=\sum_{\mathrm{U}_{l} \in \mathcal{U}} \dot{Q}_{\left(\mathrm{E}_{j}\right)}^{\left(\mathrm{U}_{l}\right)}+\sum_{\mathrm{U}_{l} \in \mathcal{U}} \dot{Q}_{\left(\mathrm{U}_{l}\right)}^{\left(\mathrm{E}_{j}\right)}+\sum_{\mathrm{E}_{j} \in \mathcal{E}} \dot{W}_{\left(\mathrm{E}_{j}\right)}^{\text {ext, in }}
$$

A compact form of the linear objective function is given in terms of the decision variables $\boldsymbol{\varphi}=(\dot{\mathbf{N}}, \dot{\boldsymbol{\Gamma}}, \dot{\mathbf{Q}}, \dot{\mathbf{W}})^{\top}$, where $\dot{\mathbf{N}}, \dot{\boldsymbol{\Gamma}}, \dot{\mathbf{Q}}, \dot{\mathbf{W}} \in \mathcal{F}$ are row vectors:

$$
f(\varphi)=\mathbf{c}^{\top} \boldsymbol{\varphi}
$$

where the entries of the cost vector $\mathbf{c}^{\top}=\left(\mathbf{c}_{\dot{\mathbf{N}}}, \mathbf{c}_{\dot{\boldsymbol{\Gamma}}}, \mathbf{c}_{\dot{\mathbf{Q}}}, \mathbf{c}_{\dot{\mathbf{W}}}\right)$ are as follows: $\mathbf{c}_{\dot{\mathbf{N}}}=\mathbf{c}_{\dot{\boldsymbol{\Gamma}}}=\mathbf{0}$ and $\mathbf{c}_{\mathbf{Q}^{\text {ext }}}=\mathbf{c}_{\mathbf{W}^{\text {ext,in }}}=1$. The resulting linear program, applying the objective function Eq. (15) and the constraints introduced in section 2.2 and 2.3 is presented in the supplementary material (Eq. (S25)). 


\section{Results}

\subsection{Comparison of sequential and simultaneous procedure}

This section emphasizes not only the influence of heat integration on the optimal pathway, but also the need of a simultaneous consideration of heat and mass flux optimization. A benchmark scenario is defined, which follows the sequential procedure, in which the flow problem is optimized without consideration of heat integration first and the heat integration potential is subsequently evaluated with the help of Pinch-based analysis (Kokossis et al., 2015, Ulonska et al., 2016, Gençer and Agrawal, 2018).

Subsequently, the FluxMax approach is applied to the same benchmark scenario. The two approaches presented in section 2.3 - direct and indirect heat integration - are compared and discussed.

\subsubsection{Benchmark scenario: A sequential procedure}

In the benchmark scenario, the energy-optimal (Eq.(15)) pathway should be identified if the feedstock - hydrogen and carbon monoxide - is fed into the process at a temperature of $25{ }^{\circ} \mathrm{C}$ and a pressure of 1 bar and the product - pure methanol - has to be delivered at $25{ }^{\circ} \mathrm{C}$ and 50 bar. Since in this first analysis the flux optimization is decoupled from heat integration, the energy duties have to be provided completely from external sources.

The elementary processes introduced in section 3.1 are used to discretize the five-dimensional thermodynamic state space (molar fractions of the components $x_{\mathrm{CO}}, x_{\mathrm{H}_{2}}, x_{\mathrm{CH}_{3} \mathrm{OH}}$; temperature $T$; and pressure $p$ ). To illustrate the results in a three-dimensional state space representation, the molar fractions of carbon monoxide and hydrogen are omitted in Fig. 5 . The TSNs corresponding to the feedstock and product are marked as a magenta and a green circle, respectively. In addition, the elementary processes are illustrated as blue thin lines connecting the discrete TSNs, illustrated as black circles. For this first analysis, the thermodynamic state space is discretized in a coarse grid (45 TSNs) to obtain a benchmark scenario that allows the comparison with direct heat integration among entities. This is because the fineness of the discretization is limited in case of direct heat integration, since the number of constraints increases drastically if the number of entities increases, as stated in section 2.3. 


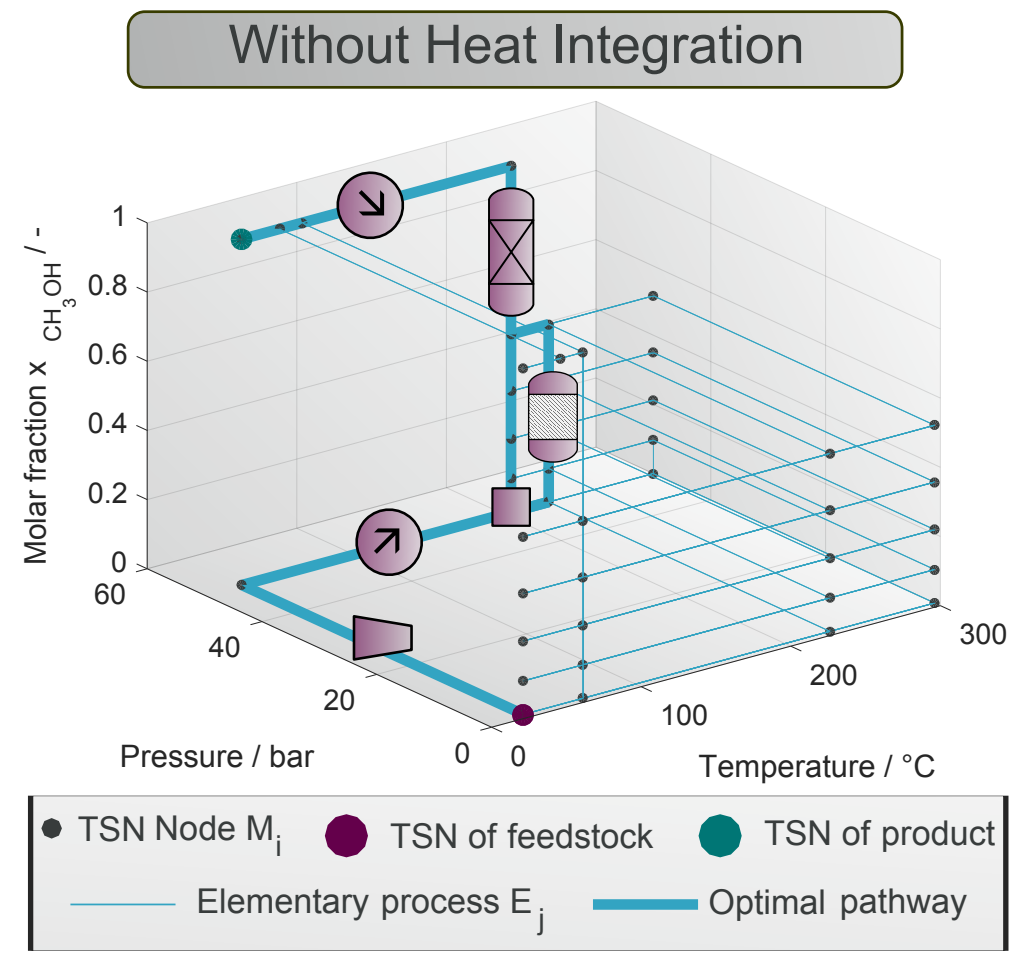

\section{External Energy Duties}

$\dot{\mathrm{Q}}_{\mathrm{in}}^{\mathrm{ext}}=1.85 \mathrm{MW}$

$\dot{Q}_{\text {out }}^{\text {ext }}=20.29 \mathrm{MW}$

$\dot{\mathrm{W}}_{\text {in }}^{\text {ext }}=5.77 \mathrm{MW}$

Heat Integration Potential

Pinch $\Delta T_{m n}=20 \mathrm{~K} \quad: 1.62 \mathrm{MW}$

Direct ${ }^{\Delta T_{m n}}=20 \mathrm{~K} \quad: 1.62 \mathrm{MW}$

Indirect $\Delta \Delta_{\mathrm{Tn}}=20 \mathrm{~K}: 0.86 \mathrm{MW}$

Indirect $\Delta \mathrm{T}_{\min }=10 \mathrm{~K}: 1.51 \mathrm{MW}$

Elementary Process Nodes

Reaction

Separation

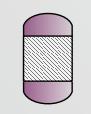

Heating

(7)

Compression

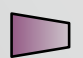

Figure 5: Optimal pathway of benchmark case in the discretized state space; corresponding elementary processes are represented along the path.

The discrete options for the reaction are two different reaction temperatures $-230{ }^{\circ} \mathrm{C}$ and $300{ }^{\circ} \mathrm{C}$ - and reactor outlet compositions $-x_{\mathrm{CH}_{3} \mathrm{OH}}: 0.10,0.21,0.35$ and 0.51 (if within the feasible region depicted in Fig. 4) - leading to different separation tasks. Depending on the pressure of the different separation inlet compositions resulting from different reaction outlet compositions, the separation temperature is set to the corresponding boiling temperature of methanol (Fig. 4 B). To adjust the required temperature and pressure levels, heating and cooling as well as compression and expansion between TSNs are considered, as illustrated in Fig. 5 with thin blue lines along the temperature and pressure axis, respectively.

The optimal path (bold blue line) within the thermodynamic state space for the benchmark scenario is shown in Fig. 5. The different elementary processes - reaction, separation, heating, cooling, compression, and mixing - are also assigned to the corresponding path. First, the reactants - hydrogen and carbon monoxide - are mixed under ambient conditions. The mixing itself, however, is not visible in the state space representation of Fig. 5, since 
only the molar fraction of the product is depicted, which does not allow a differentiation of the individual TSNs of the raw materials. The feedstock mixture is then compressed to a pressure of 50 bar and heated to the reaction temperature of $230{ }^{\circ} \mathrm{C}$. The EPN at lower temperature of $230{ }^{\circ} \mathrm{C}$ is selected because less heating - of the feedstock - and cooling of the reaction and products - is required compared to a reaction at $300{ }^{\circ} \mathrm{C}$. The reaction is performed up to the maximum amount of about $51 \%$ methanol, as the total energy requirement - heating, cooling, and power - is smaller than for the reactor outlet with a lower methanol content. In this case, either the unconverted reactants would have to be reheated to the reaction temperature after product separation - resulting in increased heating demand - or the initial feedstock flow would have to be increased - resulting in a higher heating and power demand due to increased compression demand. The reaction mixture, which contains of about $51 \%$ methanol is cooled to condensate the methanol in the separator at $205{ }^{\circ} \mathrm{C}$, which corresponds to the boiling temperature of methanol at 50 bar. While the unconverted feedstock is recycled, the pure methanol is finally cooled to the desired temperature of $25^{\circ} \mathrm{C}$.

The optimal pathway requires a total energy duty of about $27.91 \mathrm{MW}$, which is about 1.85 MW for heating, 20.29 MW for cooling, and 5.77 MW for electrical energy. Cooling and electrical energy demands are much higher than the heating demand, since the heating is only required to bring the feedstock to reactor inlet temperature, while cooling is required to cool the product, the exothermic reactor and the compressor. However, the external heating demand can still be further decreased if heat integration is taken into account. For the benchmark scenario a Pinch analysis for the identified optimal configuration was performed to evaluate the heat integration potential, resulting in a maximum internally transferable heat flux of approximately 1.62 MW.

In addition, the heat integration potential for the optimized configuration is investigated using the direct heat integration approach - among entities - and the indirect heat integration approach - using utilities, introduced in section 2.3. The comparison of the Pinch result with the prediction of our method shows that the consideration of direct heat integration lead to the same heat integration potential of $1.62 \mathrm{MW}$. In contrast, the consideration of indirect heat integration slightly underestimates the heat integration potential. Since 
heat fluxes can only be transferred to or from utilities at distinct temperature levels, the calculated internal heat flux depends on the selected temperature levels of the utilities. In addition, the desired minimum temperature difference $\Delta T_{\min }$ influences the calculation of the heat integration potential for the different methods differently. While two heat fluxes - one hot, one cold - interact directly in the classical pinch analysis and in consideration of heat integration among entities, the two heat fluxes interact indirectly via the utilities in consideration of utilities. Thus $\Delta T_{\min }$ is considered twice, because the hot flux transfers the heat in a first step to the utility - considering $\Delta T_{\min }$ - and then in a second step to the cold flux. In order to improve the comparability of all presented heat integration methods, the calculated internal heat fluxes for the case of considering utilities are not only presented for $\Delta T_{\min }=20 \mathrm{~K}$, but also for $\Delta T_{\min }=10 \mathrm{~K}$, which leads to heat integration potentials of $0.86 \mathrm{MW}$ and $1.51 \mathrm{MW}$, respectively.

Since the elementary processes in the thermodynamic state space represent unit operations, the optimal process configuration can also be illustrated as a flowsheet of process units. Fig. 6 A shows the optimal process configuration of the benchmark scenario identified by a sequential procedure. The process units - mixer, compressor, heater/cooler, reactor, and separator - are connected by mass fluxes, which are represented as black arrows. The heat fluxes are represented by red arrows and the work fluxes by blue arrows. The thickness of the red and blue arrows corresponds to the amount of the energy flux, represented by the corresponding arrow. A thick arrow indicates a high amount energy required or released, while a thin arrow indicates a low amount of energy. As the orientation of the arrows denotes the direction of the fluxes, it can be see that electrical energy is required only for the operation of the compressor and heating only for preheating the reactor inlet stream, which consists of initially provided reactants and unconverted reactants separated and recycled from the reactor outlet. The heat integration potential - identified by the classical Pinch analysis and by the proposed direct and indirect method - results from the possibility to partially utilize the excess heat of the reactor. The resulting heat flux distributions are shown in Fig. 6 B and C. It is evident that in both cases a part of the excess heat of the reactor is used to preheat the reactants. The first heater, which heats the reactants to the temperature of 
$205{ }^{\circ} \mathrm{C}$ - corresponding to the mixing point of initial reactants and recycled, unconverted reactants - is completely fed by the internal heat flux and the heat demand of the second heater, which heats the reactants to reaction temperature, is reduced by about $89.0 \%$ for direct heat integration and by about $86.5 \%$ for indirect heat integration. This results in overall percentage reduction in external heat duty of $90.3 \%$ and $87.4 \%$, respectively.

While direct heat integration - Fig. $6 \mathrm{~B}$ - uses the excess heat of the reactor directly to preheat the feedstock, indirect heat integration - Fig. $6 \mathrm{C}$ - uses a network of utilities. According to Pinch analysis, the external heat fluxes are provided at the maximum temperature and released at the minimum temperature. This concept of using excess heat of the reactor to preheat the feed is also applied in reality and is referred to as feed heat exchanger (FEHE) (Dimian, 2008, Jogwar and Daoutidis, 2015, Dimian and Bildea, 2018).

The first important result is that the FluxMax approach is able to identify the wellknown and and widely used concept of FEHE if applied in a sequential procedure. However, the strength of the FluxMax approach lies in its ability to simultaneously optimize the flow problem while taking into account heat integration, which can lead to new, non-intuitive, process designs. Therefore, the simultaneous approach will be examined in more details below.

\subsubsection{FluxMax approach: A simultaneous approach}

With the benchmark scenario defined above, the influence of simultaneous consideration of heat integration as an integrated part of the overall optimization problem is investigated. In addition, the advantages and limitations of the two proposed methods - direct and indirect - heat integration are presented and discussed.

Fig. 7 shows the optimal pathways for the benchmark scenario considering the two heat integration methods presented in section 2.3. When analyzing the results, two things are apparent. First, the consideration of direct and indirect heat integration identifies identical process configuration. And secondly, the newly identified process configuration differs from the optimal process configuration identified in the sequential procedure (Fig. 5) of decoupling the flux optimization from the determination of the heat integration potential. 


\section{(A) No Heat Integration}

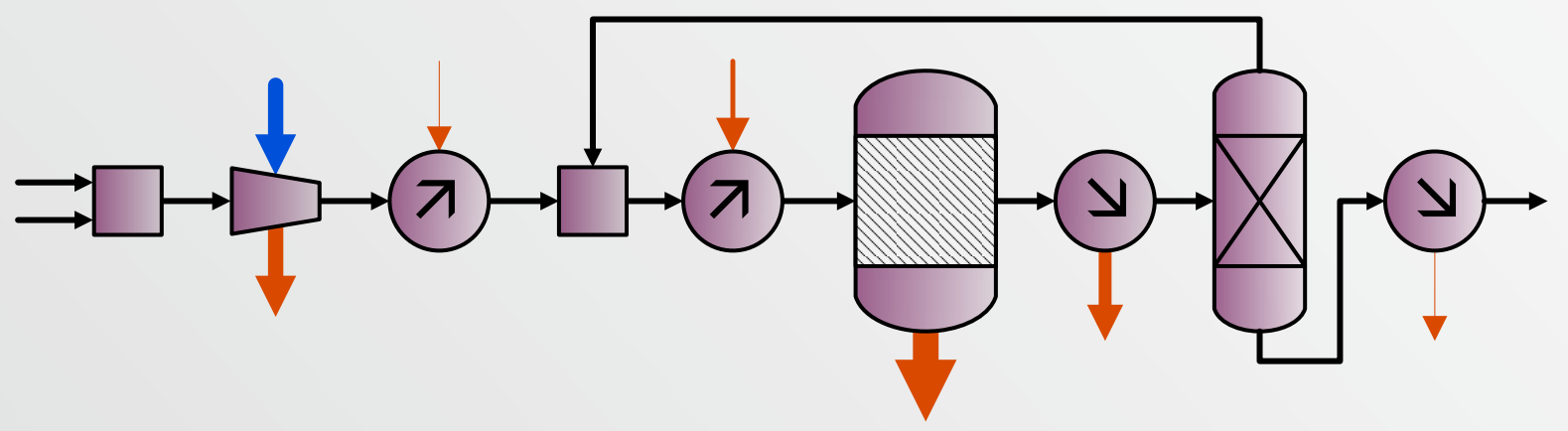

(B) Direct Heat Integration Potential

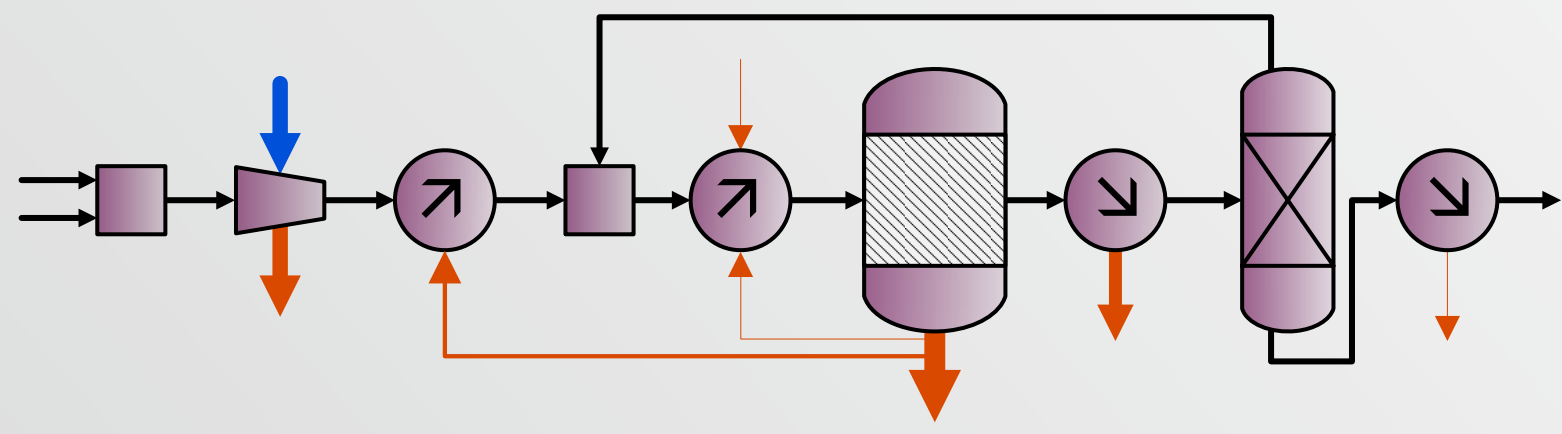

(C) Indirect Heat Integration Potential

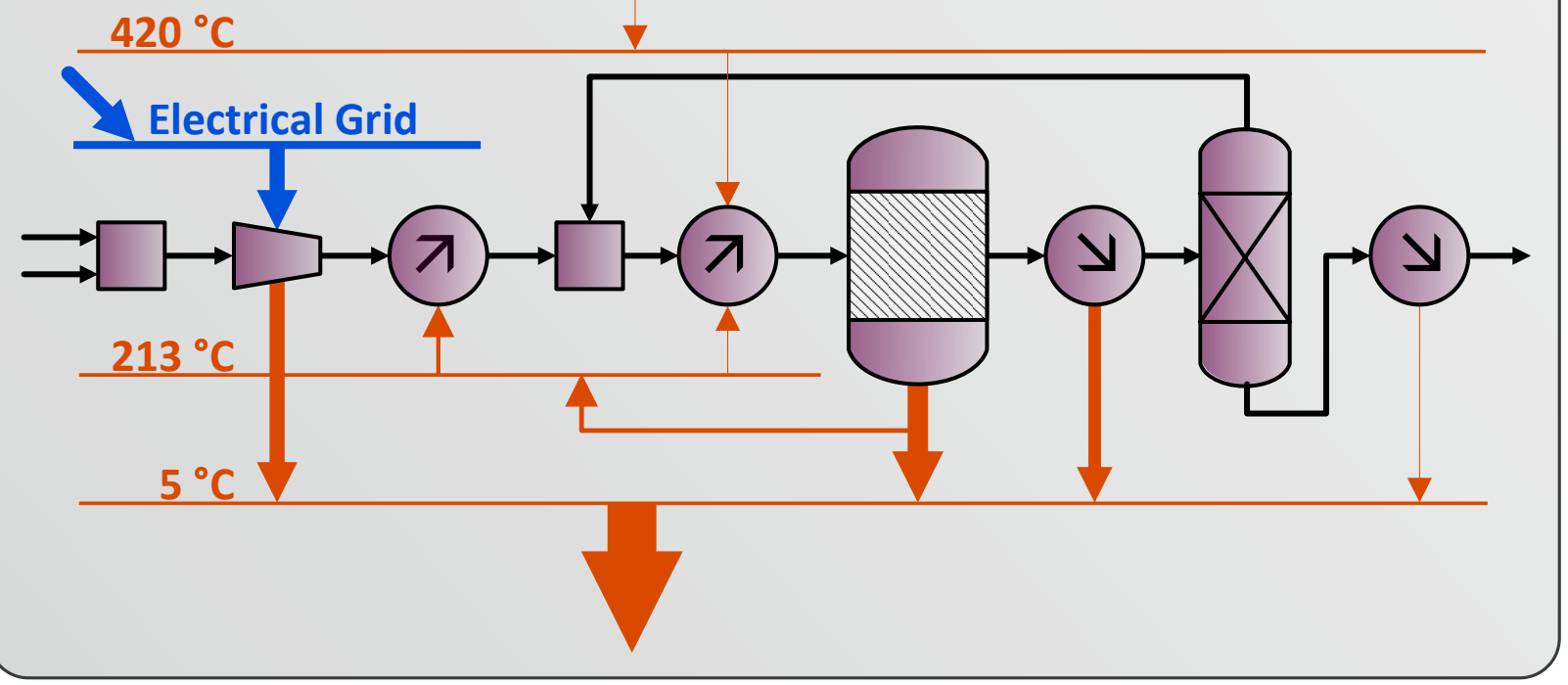

Figure 6: Schematic illustration of the optimal process configurations of the benchmark scenario obtained in a sequential procedure; optimal flowsheet if no heat integration is taken into account (A); additionally the heat integration potentials for direct $(\mathrm{B})$ and indirect heat integration $(\mathrm{C})$ are shown. 


\section{A) Direct Heat Integration}

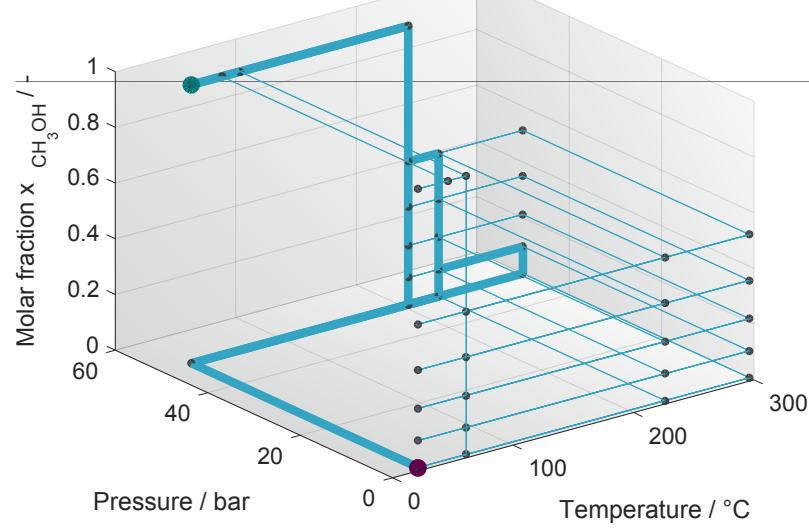

TS Node $\mathrm{M}_{\mathrm{i}}$
TSN of feedstock

\section{C}

TSN of product
B) Indirect Heat Integration

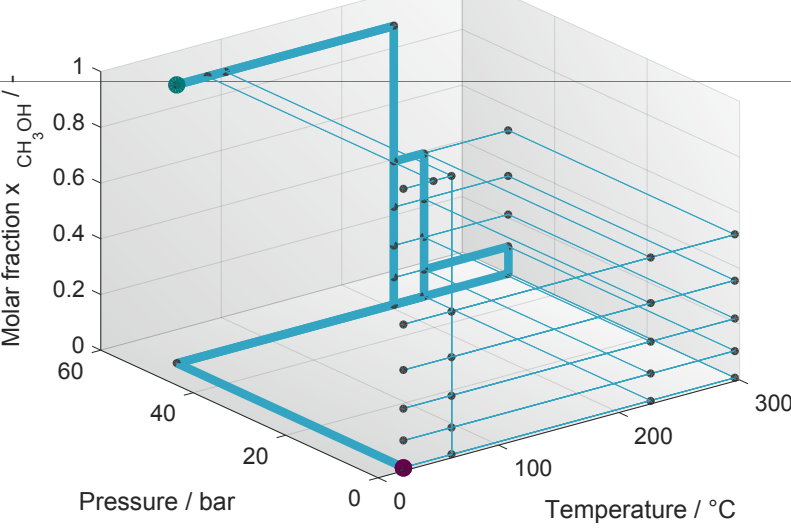

Elementary process $\mathrm{E}_{\mathrm{j}}$ Optimal pathway

\section{Heat Integration Potential}

Pinch $\Delta T_{\min }=20 \mathrm{~K} \quad: 1.92 \mathrm{MW}$

Direct $\Delta \mathrm{T}_{\min }=20 \mathrm{~K} \quad: \quad 1.93 \mathrm{MW}$

Indirect $\Delta \mathrm{T}_{\min }=20 \mathrm{~K}: 1.47 \mathrm{MW}$

Indirect $\Delta \mathrm{T}_{\min }=10 \mathrm{~K}: 1.91 \mathrm{MW}$

Figure 7: Optimal pathway in discretized state space obtained in a simultaneous procedure; optimal pathways for direct (A) and indirect heat integration (B); in addition, external energy demands and heat integration potentials are given. 
In the newly identified optimal configuration, the reaction is not only carried out at the lower temperature of $230{ }^{\circ} \mathrm{C}$, but also in a parallel reaction at the higher temperature of $300{ }^{\circ} \mathrm{C}$, since in this way the total energy demand is minimized. As the chemical equilibrium is shifted towards reactants at higher temperatures, however, the reaction is only carried out to a molar methanol content of $10 \%$ at elevated temperature up. Although the net heating demand is increased by the additional preheating of the reactants, an even higher amount of heat resulting from the excess heat of the reactor at $300{ }^{\circ} \mathrm{C}$ and the cooling of the reactor outlet can be integrated internally. The consideration of direct heat integration leads to a similar total energy requirement of about 24.24 MW (0.016 MW for heating, 18.45 MW for cooling, and 5.77 MW electrical energy) as the consideration of indirect heat integration. The following energy duties are calculated depending on the minimum temperature difference $\Delta T_{\min }$ i) for $\Delta T_{\min }=20 \mathrm{~K}: 0.479 \mathrm{MW}$ for heating, $18.92 \mathrm{MW}$ for cooling, and $5.77 \mathrm{MW}$ electrical energy, and ii) for $\Delta T_{\min }=10 \mathrm{~K}: 0.043 \mathrm{MW}$ for heating, $18.48 \mathrm{MW}$ for cooling, and 5.77 MW electrical energy. While the total energy duty is dominated by the cooling of the reactor and the electrical energy demand, which is the same as for the benchmark case, the heating plays only a minor role for the methanol synthesis process. Compared to the benchmark case, this only leads to a small improvement in the heating demand reduction. However, it has been shown that the FluxMax approach identifies new process configurations when heat integration is considered as a part of the flux optimization. To validate the obtained results, a common Pinch analysis is applied to the novel configuration leading to a heat integration potential of $1.92 \mathrm{MW}$. A slight overestimation of the Pinch result for the direct heat integration $(1.93 \mathrm{MW})$ and a slight underestimation for the indirect heat integration (1.47 MW or 1.91 MW, respectively) is observed. The overestimation was also observed in (Schack et al., 2017) and is present when two or more hot entities partially provide heat to a cold entity. The reason for this is that the inqualities (Eqs. (7) and (9)) consider only the initial temperatures and not the actual temperatures that could due to already internally integrated heat fluxes.

Though, the heat integration potential calculated with the Pinch analysis also shows an increase in the internally transferable heat fluxes and thus a decrease in externally provided 
energy for the new configuration compared to the benchmark configuration as shown in Tab. 2. This is a very important result as it underlines the need for a simultaneous procedure and the ability of the FluxMax approach to identify globally optimal process configurations. In particular, if the energy duty depends stronger on the heating duty than in the case scenario under consideration, the FluxMax approach can be a powerful tool for designing new, non-intuitive, processes (Liesche et al., 2019). The saving potentials listed in Tab. 2 highlight the latter statement. While the FluxMax approach enables the identification of process structures that almost completely exploit the existing heat integration potential (approx. $99 \%$ compared to $88 \%$ ), the total energy savings for the considered case study are only slightly increased from approx. $11 \%$ to approx. $13 \%$, since the external cooling duty remains high after exploitation of the heat integration potential.

Table 2: Overview of the external heating and cooling duties of the sequential and simultaneous approach and corresponding saving potentials.

\begin{tabular}{llllllll}
\hline Energy flux & No HI & \multicolumn{3}{c}{ Sequential } & \multicolumn{3}{c}{ Simultaneous } \\
& & direct & indirect Pinch & direct & indirect Pinch \\
\hline $\begin{array}{c}\text { External duties } \\
\text { external heating / MW }\end{array}$ & 1.850 & 0.230 & 0.340 & 0.230 & 0.016 & 0.043 & 0.026 \\
$\quad \begin{array}{l}\text { external cooling / MW } \\
\text { electrical work / MW }\end{array}$ & 20.290 & 18.670 & 18.780 & 18.670 & 18.450 & 18.480 & 18.460 \\
$\quad$ total energy / MW & 27.910 & 5.770 & 5.770 & 5.770 & 5.770 & 5.770 & 5.770 \\
Savings & & 23.960 & 24.890 & 24.670 & 24.236 & 24.293 & 24.256 \\
$\quad$ saving in total energy / \% & 0 & 11.6 & 10.8 & 11.6 & 13.2 & 13.0 & 13.1 \\
$\quad$ saving in heating / \% & 0 & 87.6 & 81.6 & 87.6 & 99.1 & 97.7 & 98.6 \\
\hline
\end{tabular}

The optimum that is identified for indirect heat integration depends on the number of utilities considered and their temperature levels. The dependency of the number of utilities on the result is analyzed in section 4.2. It can be stated, however, that even a coarse discretization was able to identify a configuration with increased internally integrated heat fluxes. As a consequence, only the indirect heat integration approach is used in the following, since the computational effort is significantly lower compared to the direct approach, which allows a finer discretization of the thermodynamic state space.

Fig. 8 shows the flowsheet representation for the optimal trajectory found in the ther- 


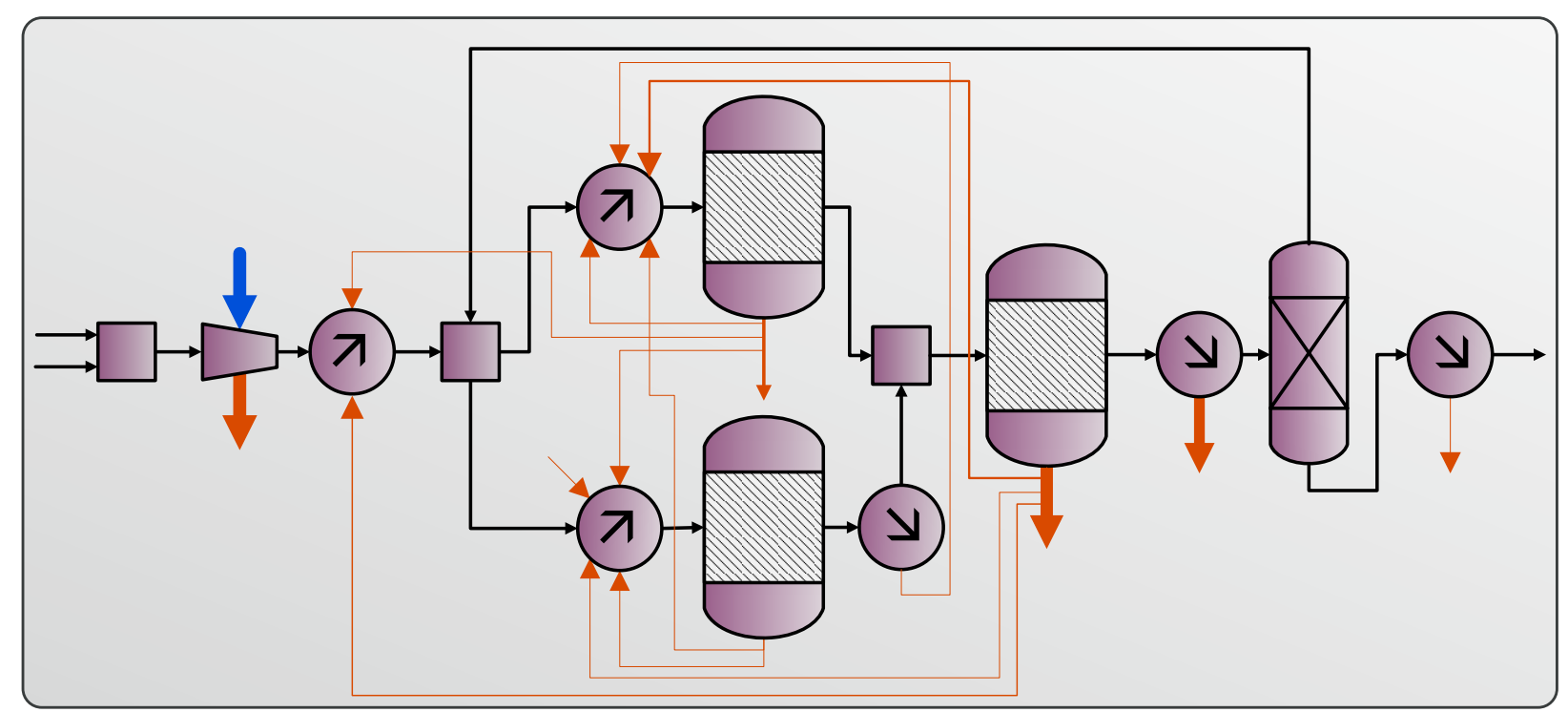

Figure 8: Schematic illustration of the optimal process configurations obtained in a simultaneous procedure; for better clarity, only the flowsheet is shown, taking direct heat integration into account.

modynamic state space. For better visibility the energy flux distribution resulting from consideration of indirect heat integration is omitted and only the flowsheet with direct heat integration is displayed. However, the following statements on Fig. 8 also apply to the case of indirect heat integration, since the general flowsheets are interchangeable. The parallel reaction at an elevated temperature requires an additional heater to provide the reactants at higher reaction temperatures and an additional cooler to cool the reaction outlet - with a methanol content of about $10 \%$ - to the reaction temperature of the first reactor to be further converted at lower temperature. Besides this change in the reaction part of the process, the other process units correspond to the configuration of the benchmark process, shown in Fig. 6.

It can be seen that the excess heat of the parallel reactor at higher temperature and of the new cooler is completely integrated internally. In this way, the reactants entering the first reactor, can be better preheated by internal heat fluxes.

\subsection{Optimal utility network}

The FluxMax approach guarantees the identification of the global optimum if a convex objective function is used, because the constraints are linear in terms of the decision variables 
as a direct result of the formulation of the FluxMax approach. However, the result depends strongly on the selected discretization of the thermodynamic state space, as da Cruz and Manousiouthakis (2017) has shown in their discretization studies. In the previous section, a coarse grid was used to better visualize the results and reduce the computational time - in particular when applying the direct heat integration approach. In this section, the effect of the discretization of utility temperatures of the indirect heat integration approach is analyzed.

The internally transferred heat depends strongly on the a priori defined temperature levels of the considered utilities. Since external energy fluxes are also provided or released via utilities for the indirect heat integration approach, the minimum number of utilities equals two: one utility at a sufficiently low temperature to provide the external cooling and one utility at sufficiently high temperature to provide the external heating, respectively. Fig. 9 illustrates the external heat duty for the scenario introduced above as a function of number of utilities considered. The temperatures of the utilities are equidistantly distributed between $5{ }^{\circ} \mathrm{C}$ and $420{ }^{\circ} \mathrm{C}$ and are listed in Tab. S2.

For the consideration of only two utilities no internal heat transfer is possible and external heat duty corresponds to the heat duty calculated for the benchmark process (see also Fig. 5). If one considers utilities at a temperature between these outer limits, the external heating duty is reduced as a result of the internal heat integration potential. It is evident, however, that an increase of considered utilities does not necessarily mean a decrease in external heat duty. The reason for this is that not (only) the number of considered utilities, but also the particular temperature is decisive for a high heat integration potential. Due to the equidistant distribution of the temperature levels considered an additional utility affects all the other temperatures of remaining utilities. In other words: While the probability of a higher heat integration potential estimated by the indirect approach increases with an increased number of utilities considered, even a low number of utilities can lead to a maximum heat integration potential.

For the scenario defined above, an optimal number of seven utilities is found, resulting in the external heat duty of $43.32 \mathrm{~kW}$. But also the consideration of only four utilities leads 


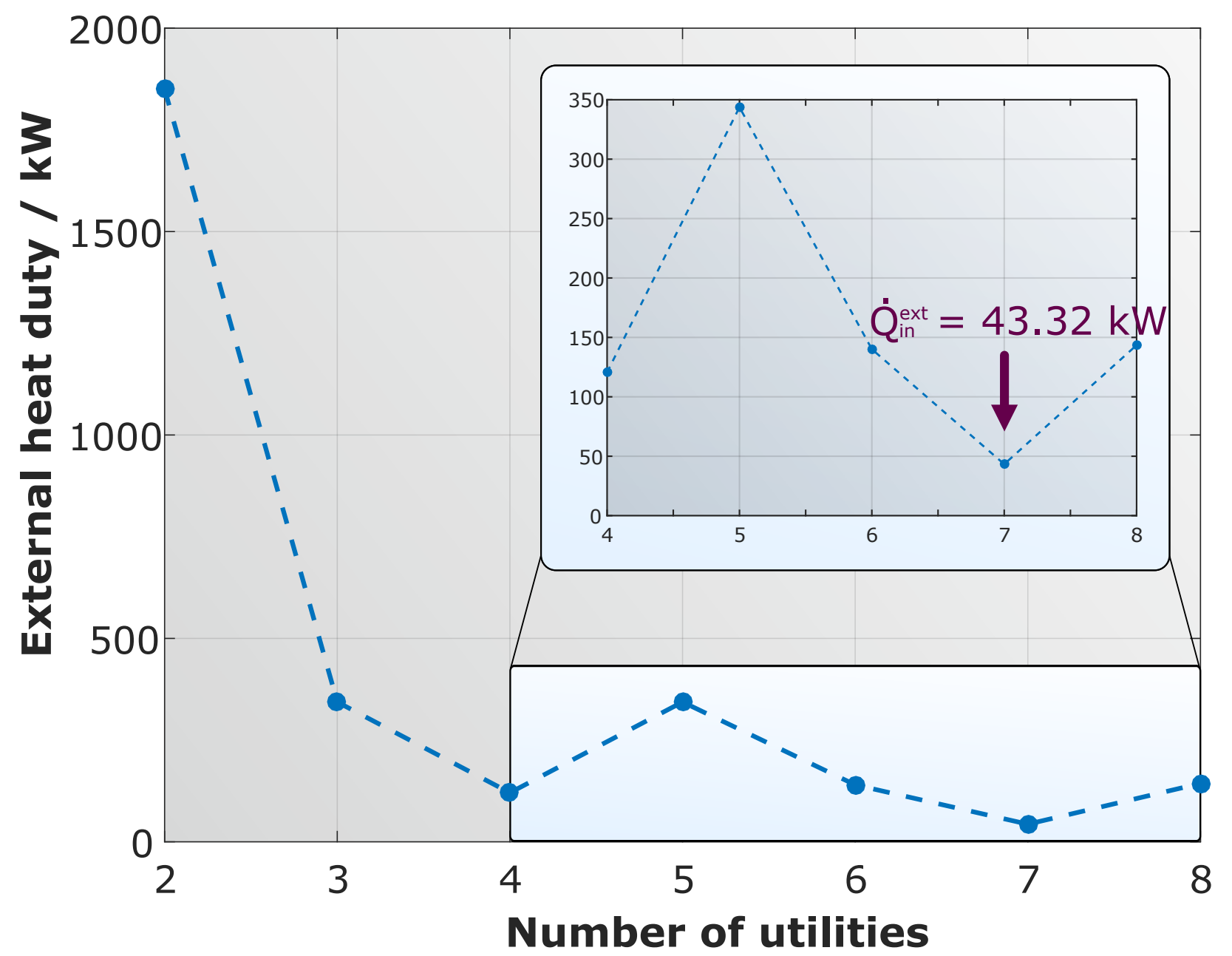

Figure 9: Illustration of the external heating requirement as a function of the number of utilities considered. 
to an acceptable result. Since an increase in the number of utilities considered could lead to an increase in costs - which are not considered in this study - the choice of the actual number of utilities considered in a real world application may differ. However, in this study - both for the previously presented results and for the subsequent results - the number of utilities considered is set to seven, as listed in Tab. 3 .

Table 3: Optimal number of utilities and corresponding temperatures used in the study.

\begin{tabular}{lccccccc} 
Utility & 1 & 2 & 3 & 4 & 5 & 6 & 7 \\
Temperature $/{ }^{\circ} \mathbf{C}$ & 5 & 74 & 143 & 213 & 282 & 351 & 420 \\
\hline
\end{tabular}

In addition to illustrating the impact of the number of utilities, the results presented demonstrate another key feature of the FluxMax approach: the optimization of utility networks. In the case study under consideration, the optimizer selects three utilities $-5{ }^{\circ} \mathrm{C}$, $213{ }^{\circ} \mathrm{C}$, and $420{ }^{\circ} \mathrm{C}$ - from the options listed in Tab. 3. Thus, the FluxMax generally enables the identification of the optimal temperature levels of the utilities by introducing a multitude of utility nodes, which provide heating or cooling at different temperatures. Particularly when considering distinct cost for the external heat fluxes to provide heating or cooling at distinct temperature levels, further interesting optimization tasks are facilitated.

\subsection{Identification of optimal process designs}

In the previous sections, the FluxMax approach was applied to a coarse discretization of the thermodynamic state space. In this way, key features - such as the simultaneous consideration of heat integration - could be demonstrated while maintaining an intuitive understanding of the obtained results. In this section, the focus is on the process optimization, which requires a finer discretization of the thermodynamic state space.

The discretization of the whole thermodynamic state space is not done equidistantly, since there is a distinct operation window for each elementary process. The methanol synthesis reaction is normally performed between temperatures of $230{ }^{\circ} \mathrm{C}$ and $300{ }^{\circ} \mathrm{C}$ and in a pressure range of 50 bar to 100 bar (Ott et al., 2000). Therefore, the reaction conditions were discretized within the technically applied range: the pressure at which the reaction can take place in steps of 10 bar and the temperature in steps of $10{ }^{\circ} \mathrm{C}$. The extent of reaction 
was discretized in such a way that discrete molar fractions of methanol between zero and chemical equilibrium - in 0.06 steps - were achieved. To separate the reactor outlet, the product stream must be cooled to the condensation temperature of methanol to be flashed into liquid methanol and gaseous, unconverted reactants. The condensation temperatures as a function of the pressure is calculated by the Clausius Clapeyron equation (Eq. (S11) in the supplementary material). The resulting discretized thermodynamic state space (810 TSNs) and possible elementary processes, that connect the TSNs, are shown in the supplementary material in Fig. S2 .

For the identification of process pathways that optimally convert the feedstock into the desired product specifications, only indirect heat integration was considered in order to enable an finer discretization. The number of utility nodes, that provide the external energy duties and enable the internal heat transfer, is set to seven according to Table 3.

The initial reactants are supplied at ambient conditions $-25^{\circ} \mathrm{C}$ and 1 bar - and an annual production of $100,000 \mathrm{t}_{\mathrm{CH}_{3} \mathrm{OH}}$ /a pure methanol is desired. Two different product specifications are examined: case A) ambient target conditions of the product $\left(T_{\operatorname{targ}}=25^{\circ} \mathrm{C}\right.$, $\left.p_{\text {targ }}=50 \mathrm{bar}\right)$; and case B) elevated target temperature and pressure of the product $\left(T_{\operatorname{targ}}=\right.$ $\left.150{ }^{\circ} \mathrm{C}, p_{\operatorname{targ}}=100 \mathrm{bar}\right)$. The specifications of case A are the same as for the benchmark scenario. In case $\mathrm{B}$, the methanol is desired to be delivered at increased temperature and pressure, which may correspond to the case, that methanol is not the final product but an intermediate, that needs to be further processed.

The optimal pathways in the discretized thermodynamic state space are illustrated in Fig. 10. In contrast to previously presented results, the connections between TSNs, that represents the possible elementary process functions, are omitted for better readability. The optimal trajectory is again depicted as blue line.

Before analyzing the two cases in detail, it can be seen that the desired product specification only affects the downstream part of the process. Both cases have in common that the initial reactants are mixed, then compressed to the lowest possible reaction pressure of 50 bar and heated to be converted in two parallel reactors. Most of the reaction is performed at the lowest possible reaction temperature of $230{ }^{\circ} \mathrm{C}$, while a second reactor is performed 


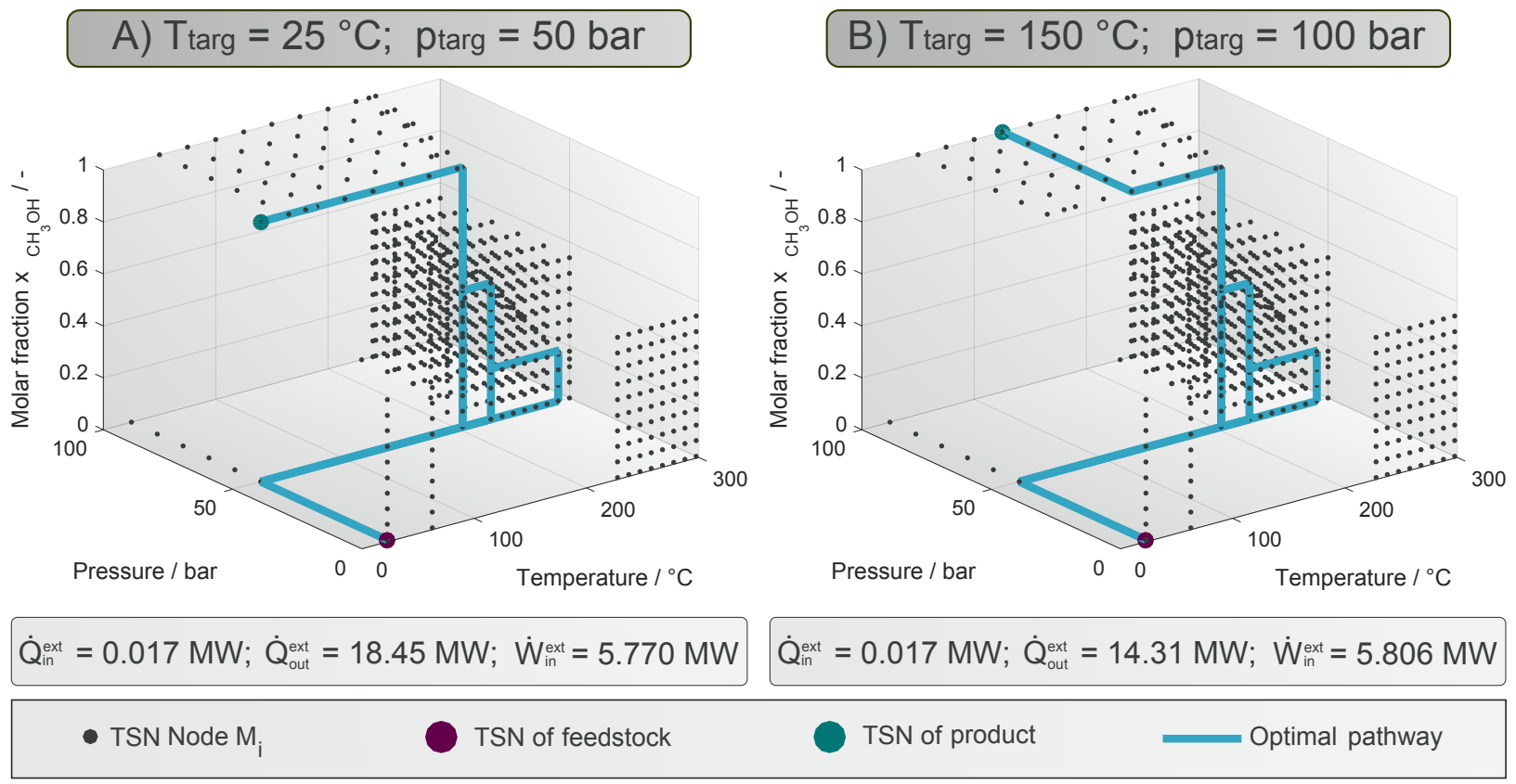

Figure 10: Optimal pathway in discretized state space for cases A) $T_{\operatorname{targ}}=25^{\circ} \mathrm{C}, p_{\operatorname{targ}}=50$ bar and B) $T_{\text {targ }}=150{ }^{\circ} \mathrm{C}, p_{\text {targ }}=100$ bar.

at evaluated temperature of $290{ }^{\circ} \mathrm{C}$. The parallel reaction at evaluated temperature allows the utilization of the excess reactor heat fluxes to preheat the reactants as described in section 4.1 and shown in Fig. 8. Both reactions are carried out to the maximum extent at the corresponding temperature (Fig. 4), resulting in a methanol outlet fraction of the low temperature reactor of $x_{\mathrm{CH}_{3} \mathrm{OH}}$ of about $52.5 \%$ and of about $19 \%$ of the high temperature reactor. While in this case the achieved reactor outlet fractions correspond to the chemical equilibrium composition, it is important to mention that the FluxMax approach in general also facilitates the use of kinetic reactor models, as shown in Liesche et al. (2019). The high temperature reactor outlet stream is cooled and further converted in the low temperature reactor. Subsequently, the overall reactor outlet stream is cooled to meet the condensation temperature to separate the unconverted reactants. The unconverted reactants are recycled, while the pure methanol is brought to the desired product specification in a final step.

Case A results in almost the same optimal configuration that was found when the FluxMax approach was applied to the benchmark case (Fig. 7). Due to the increased discretization, however, the parallel reaction is performed at a slightly lower temperature of $290^{\circ} \mathrm{C}$. 
As can be seen in Fig. 10, the energy duties can be reduced further in this way, because the amount of external heat duty to preheat the reactant inlet stream of the second reactor is smaller compared to the benchmark process. The finer discretization of the elementary reaction process improves the identified optimum. The improvement is very small yet, thus in this study an even finer discretization was omitted. However, an optimization on an finer grid would be possible without problems.

The case B differ in the desired target temperature and the target pressure, which requires additional compression, resulting in increased power consumption. Although the excess heat of compression must be cooled, the overall cooling duty is smaller compared to case A, because the product only has to be cooled to $150^{\circ} \mathrm{C}$. Interestingly, however, the reaction is carried out at a pressure of 50 bar not directly at target pressure of 100 bar. The reason is that compression of one mole of liquid methanol requires less power than compression of three moles of gaseous reactants -1 mole of $\mathrm{CO}$ and 2 mole of $\mathrm{H}_{2}$.

The presented results demonstrate that the FluxMax approach can be used for process design tasks by optimizing the mass- and energy fluxes. Once the thermodynamic state space is discretized and elementary process defined, the desired reactant and product specifications are easily adjustable. As a result, the FluxMax approach is very versatile in the analysis and optimization of different case scenarios. Although this study focuses only on the identification of energy-optimal processes, in general any objective function could be applied to the FluxMax. In previous studies, at which the FluxMax approach is based on, we have already used different objective functions that could be directly applied to the FluxMax approach: for the optimization of chemical production networks, minimizing costs and $\mathrm{CO}_{2}$-emission (Schack et al., 2016, 2018), and for the methanol synthesis process, minimizing capital cost of the compressor cascade and maximizing the kinetic rates of the reactor part (Liesche et al., 2018).

\section{Conclusions}

In this paper we presented the FluxMax approach for process design and synthesis under consideration of heat integration by discretization of the thermodynamic state space. The 
introduction of thermodynamic state nodes (TSN), elementary process nodes (EPN), utility nodes (UN), and work utility nodes (WUN) enables the representation of the chemical process as a directed graph, with the edges corresponding to the mass and energy fluxes to be optimized. All mixtures in the process are uniquely determined by thermodynamic coordinates and thus assigned to a distinct TSN. The EPNs facilitate the thermodynamic state change between the TSNs. Therefore each elementary process is described uniformly. By introducing a generalized process extent number, a stoichiometric equation is formulated for each type of elementary process. The generalized process extent number is also used to formulate a continuous flux optimization problem that identifies the optimal pathway within the discretized thermodynamic state space. The discretization of the thermodynamic state space effectively decouples the process based nonlinearities from the network optimization problem, which result in a linear feasible region. By adding additional inequality constraints, heat integration is considered as integrated part of the flux optimization.

We have applied the FluxMax approach to the methanol synthesis process, which is of great significance for applications in the field of Renewable-to-Chemicals. A linear objective function - minimizing total energy demand - was used, resulting in a purely linear optimization problem. It was shown that the FluxMax approach identifies energy-optimal process configurations that outperform configurations identified in a sequential procedures, which highlights the importance of a simultaneous approach. The complexity of the optimization problem was drastically reduced by the introduction of an indirect heat integration approach. The validation with classical Pinch analysis proved the applicability of the FluxMax approach to identify novel, non-intuitive process configurations. Furthermore, the possibility of optimizing the utility network resulting directly from the introduction of utility nodes was demonstrated.

In addition to the key features of the simultaneous consideration of heat integration and the unified representation of any chemical process as directed graph by introducing generalized stoichiometric equations, the FluxMax approach has further important aspects: The FluxMax approach is independent of the considered process scale. The EPNs can correspond to: i) whole processes for the optimization of chemical production networks on production 
system level, ii) process units for the optimization of chemical processes on plant level, or iii) elementary processes for the optimization of process units. It is also possible to overlap different scales by using rigorous models to describe elementary processes of particular interest, while lumped models are used for less important elementary processes. Therefore, the FluxMax approach is a powerful tool that identifies optimal, non-intuitive, process pathways and process configurations. Especially if the underlying models are strongly nonlinear, the challenges of classical nonlinear optimization approaches could be overcome at the price of a solution which is dependent on the discretization of the thermodynamic state space.

This study showed also the necessity for further work: i) not only the fineness of the discretization but also the way of discretization - equidistantly, distributed, or adaptively refined - should be examined as the discretization plays such a major role in the accuracy of the result obtained, and ii) in this study only shortcut models were used, but the strength of the FluxMax approach is the possibility to use also sophisticated models to determine the elementary processes; and iii) the application of the FluxMax approach to distillation processes will have to be investigated to optimize the energy-intensive downstream part of the methanol synthesis process in more detail.

\section{Acknowledgments}

The author Georg Liesche is also affiliated to the International Max Planck Research School (IMPRS) for Advanced Methods in Process and Systems Engineering (Magdeburg).

\section{Nomenclature}

\section{Acronyms}

EPF Elementary Process Function

EPN Elementary Process Node

FEHE Feed heat exchanger

FMA FluxMax Approach 
LP Linear programming

${ }_{817} \Delta_{R} h^{\ominus}$ Standard enthalpy of reaction $/ \mathrm{kJ} / \mathrm{mol}$

${ }_{818} \dot{\boldsymbol{\Gamma}} \quad$ Vector of process extent numbers

${ }_{819} \dot{\Gamma} \quad$ Process extent number $/ \mathrm{mol} / \mathrm{s}$

${ }_{820} \dot{\xi} \quad$ Extent of reaction $/ \mathrm{mol} / \mathrm{s}$

${ }_{821} \quad \eta \quad$ Efficiency factor of work consumption / -

${ }_{822} \omega \quad$ Molar work duty of an EPN / kJ $/ \mathrm{mol}$

823

824

Molar heat duty of an EPN / kJ/mol

Thermodynamic coordinate of $z$-th dimension 
Latin Symbols

${ }_{826} \dot{\mathbf{N}} \quad$ Vector of mass fluxes

${ }_{827} \dot{\mathrm{Q}} \quad$ Vector of heat fluxes

${ }_{828} \dot{\mathbf{W}} \quad$ Vector of work fluxes

${ }_{829} \quad \dot{N} \quad$ Mass flux / mol/s

${ }_{830} \dot{Q} \quad$ Heat flux / kW

${ }_{831} \dot{W} \quad$ Work flux / $\mathrm{kW}$

${ }_{832}$ A Coefficient matrix of constraints

${ }_{833}$ b Vector of right-hand sides

${ }_{834}$ c Vector of cost factors

$835 \mathbf{X} \quad$ Vector of molar fractions

${ }_{836} p \quad$ Pressure / Pa

${ }_{837} T$ Temperature / K

${ }_{838} x \quad$ Molar fraction / -

839 C Cold stream

${ }_{840} \quad \mathrm{E}_{j} \quad$ Elementary process node $j$

${ }_{841} \mathrm{f} \quad$ Objective function

${ }_{842} \mathrm{H} \quad$ Hot stream

${ }_{843} \quad \mathrm{M}_{i} \quad$ Thermodynamic substance node $i$

${ }_{844} \mathrm{~S}_{k} \quad$ Work utility node $k$ 
${ }_{845} \mathrm{U}_{l} \quad$ Utility node $l$

846 Indices

847 eq Equality

848 ext External flux

${ }_{849}$ in Inlet flux

850 int Internal flux

${ }_{851}$ iq Inequality

${ }_{852}$ lb Lower bound

853 max Maximum

854 min Minimum

855 out Outlet flux

${ }_{856} \mathrm{ub} \quad$ Upper bound

857 util Utility

\section{Other Symbols}

${ }_{859} \mathcal{A} \quad$ Set of all pure substances $\alpha$

${ }_{860} \mathcal{E} \quad$ Set of elementary process nodes $\mathrm{E}_{j}$

${ }_{861} \mathcal{F} \quad$ Set of all fluxes

${ }_{862} \mathcal{K} \quad$ Set of all permutations of internally heat transferring streams

${ }_{863} \mathcal{M}$ Set of all thermodynamic substance nodes $\mathrm{M}_{i}$

${ }_{864} \mathcal{U} \quad$ Set of utility nodes $\mathrm{U}_{l}$ 


\section{References}

Bertau, M., Offermanns, H., Plass, L., and Schmidt, F. Methanol: The Basic Chemical and Energy Feedstock of the Future: Asinger's Vision Today. Springer Berlin Heidelberg, Berlin, Heidelberg, 2014. ISBN 9783642397097.

Cabezas, H., Argoti, A., Friedler, F., Mizsey, P., and Pimentel, J. Design and engineering of sustainable process systems and supply chains by the p-graph framework. Environmental Progress \& Sustainable Energy, 37(2):624-636, 2018. ISSN 19447442. doi: 10.1002/ep.12887.

Colberg, R. D. and Morari, M. Area and capital cost targets for heat exchanger network synthesis with constrained matches and unequal heat transfer coefficient. Computers \& Chemical Engineering, 14(1): 1-22, 1990. ISSN 00981354.

da Cruz, F. E. and Manousiouthakis, V. I. Process intensification of reactive separator networks through the ideas conceptual framework. Computers \& Chemical Engineering, 105:39-55, 2017. ISSN 00981354. doi: 10.1016/j.compchemeng.2016.12.006.

Dimian, A. and Bildea, C. Energy efficient methanol-to-olefins process. Chemical Engineering Research and Design, 131:41-54, 2018. doi: 10.1016/j.cherd.2017.11.009.

Dimian, A. C. Chemical process design computer-aided case studies. 2008.

Dowling, A. W. and Biegler, L. T. A framework for efficient large scale equation-oriented flowsheet optimization. Computers \& Chemical Engineering, 72:3-20, 2015. ISSN 00981354. doi: 10.1016/j.compchemeng. 2014.05.013.

Duran, M. A. and Grossmann, I. E. Simultaneous-Optimization and Heat Integration of Chemical Processes. Aiche Journal, 32(1):123-138, 1986. ISSN 0001-1541. doi: DOI10.1002/aic.690320114.

Elsido, C., Mian, A., and Martelli, E. A systematic methodology for the techno-economic optimization of organic rankine cycles. 4th International Seminar on Orc Power Systems, 129:26-33, 2017. ISSN 1876-6102. doi: 10.1016/j.egypro.2017.09.171.

Freund, H. and Sundmacher, K. Towards a methodology for the systematic analysis and design of efficient chemical processes. Chemical Engineering and Processing: Process Intensification, 47(12):2051-2060, 2008. ISSN 02552701. doi: 10.1016/j.cep.2008.07.011.

Friedler, F., Tarján, K., Huang, Y. W., and Fan, L. T. Graph-theoretic approaches to process synthesis: axioms and theorems. Chemical Engineering Science, 47:1973-1988, 1992. ISSN 00092509.

Gençer, E. and Agrawal, R. Toward supplying food, energy, and water demand: Integrated solar desalination process synthesis with power and hydrogen coproduction. Resources, Conservation and Recycling, 133: 331-342, 2018. ISSN 09213449. doi: 10.1016/j.resconrec.2018.01.030.

Hartono, B., Heidebrecht, P., and Sundmacher, K. Combined Branch and Bound Method and Exergy Analysis for Energy System Design. Industrial \& Engineering Chemistry Research, 51(44):14428-14437, 
2012. ISSN 0888-5885. doi: 10.1021/ie301232t.

Hentschel, B., Peschel, A., Freund, H., and Sundmacher, K. Simultaneous design of the optimal reaction and process concept for multiphase systems. Chemical Engineering Science, 115:69-87, 2014. doi: 10. 1016/j.ces.2013.09.046.

Holiastos, K. and Manousiouthakis, V. Minimum hot/cold/electric utility cost for heat exchange networks. Computers \&6 Chemical Engineering, 26(1):3-16, 2002. ISSN 0098-1354. doi: 10.1016/S0098-1354(01) 00726-8.

Holiastos, K. and Manousiouthakis, V. Infinite-dimensional state-space (IDEAS) approach to globally optimal design of distillation networks featuring heat and power integration. Industrial E Engineering Chemistry Research, 43(24):7826-7842, 2004. doi: 10.1021/ie010434i.

Huang, B., Li, Y., Gao, R., Zuo, Y., Dai, Z., and Wang, F. Simultaneous optimization and heat integration of the coal-to-sng process with a branched heat recovery steam cycle. Computers $\&$ Chemical Engineering, 117:117-128, 2018. ISSN 00981354. doi: 10.1016/j.compchemeng.2018.02.008.

International Energy Agency. Energy Efficiency 2018. Analysis and outlooks. 2018.

Jogwar, S. and Daoutidis, P. Optimal operation of an energy integrated batch reactor - feed effluent heat exchanger system. IFAC-PapersOnLine, 28(8):1192-1197, 2015. doi: 10.1016/j.ifacol.2015.09.130.

Kaiser, N. M., Jokiel, M., McBride, K., Flassig, R. J., and Sundmacher, K. Optimal Reactor Design via Flux Profile Analysis for an Integrated Hydroformylation Process. Industrial \& Engineering Chemistry Research, 56(40):11507-11518, 2017. ISSN 0888-5885 1520-5045. doi: 10.1021/acs.iecr.7b01939.

Kermani, M., Wallerand, A. S., Kantor, I. D., and Marechal, F. Generic superstructure synthesis of organic Rankine cycles for waste heat recovery in industrial processes. Applied Energy, 212:1203-1225, 2018. ISSN 03062619. doi: 10.1016/j.apenergy.2017.12.094.

Keßler, T., Kunde, C., McBride, K., Mertens, N., Michaels, D., Sundmacher, K., and Kienle, A. Global optimization of distillation columns using explicit and implicit surrogate models. Chemical Engineering Science, 197:235-245, 2019. doi: 10.1016/j.ces.2018.12.002.

Kim, J., Sen, S. M., and Maravelias, C. T. An optimization-based assessment framework for biomass-to-fuel conversion strategies. Energy \& Environmental Science, 6(4):1093-1104, 2013. ISSN 1754-5692 1754-5706. doi: $10.1039 / \mathrm{c} 3 e e 24243 \mathrm{a}$.

Kokossis, A. C., Tsakalova, M., and Pyrgakis, K. Design of integrated biorefineries. Computers \& Chemical Engineering, 81:40-56, 2015. ISSN 00981354. doi: 10.1016/j.compchemeng.2015.05.021.

König, A., Ulonska, K., Mitsos, A., and Viell, J. Optimal Applications and Combinations of Renewable Fuel Production from Biomass and Electricity. Energy \& Fuels, 33(2):1659-1672, 2019. ISSN 0887-0624 1520-5029. doi: 10.1021/acs.energyfuels.8b03790.

Liesche, G., Schack, D., Rätze, K. H. G., and Sundmacher, K. Thermodynamic Network Flow Approach for 
Chemical Process Synthesis. Computer Aided Chemical Engineering, 43:881-886, 2018. ISSN 1570-7946. doi: 10.1016/B978-0-444-64235-6.50154-6.

Liesche, G., Schack, D., and Sundmacher, K. The FluxMax approach for simultaneous process synthesis and heat integration: Production of hydrogen cyanide. AIChE Journal, 2019. ISSN 0001-1541. doi: 10.1002/aic.16554.

Linnhoff, B. and Flower, J. R. Synthesis of heat exchanger networks: I. Systematic generation of energy optimal networks. AIChE Journal, 24(4):633-642, 1978. ISSN 1547-5905. doi: 10.1002/aic.690240411.

Maußner, J., Dreiser, C., Wachsen, O., and Freund, H. Systematic model-based design of tolerant chemical reactors. Journal of Advanced Manufacturing and Processing, 1(3), 2019. doi: 10.1002/amp2.10024.

Moioli, E., Mutschler, R., and Züttel, A. Renewable energy storage via CO2 and H2 conversion to methane and methanol: Assessment for small scale applications. Renewable and Sustainable Energy Reviews, 107: 497-506, 2019. ISSN 13640321. doi: 10.1016/j.rser.2019.03.022.

Nagy, A. B., Adonyi, R., Halasz, L., Friedler, F., and Fan, L. T. Integrated synthesis of process and heat exchanger networks: algorithmic approach. Applied Thermal Engineering, 21(13-14):1407-1427, 2001. ISSN 13594311. doi: 10.1016/S1359-4311(01)00033-3.

Ott, J., Gronemann, V., Pontzen, F., Fiedler, E., Grossmann, G., Kersebohm, D. B., Weiss, G., and Witte, C. Methanol. Wiley-VCH Verlag GmbH \& Co. KGaA, 2000. ISBN 9783527306732. doi: 10.1002/ 14356007.a16_465.pub3.

Otto, A., Grube, T., Schiebahn, S., and Stolten, D. Closing the loop: captured CO2 as a feedstock in the chemical industry. Energy \& Environmental Science, 8(11):3283-3297, 2015. ISSN 1754-5692. doi: 10.1039/C5EE02591E.

Ouda, M., Hank, C., Nestler, F., Hadrich, M., Full, J., Schaadt, A., and Hebling, C. Power-to-Methanol: Techno-Economical and Ecological Insights, pages 380-409. Springer Berlin Heidelberg, Berlin, Heidelberg, 2019. ISBN 978-3-662-58006-6. doi: 10.1007/978-3-662-58006-6_17.

Papoulias, S. A. and Grossmann, I. E. A structural optimization approach in process synthesis - ii. Computers $\& 3$ Chemical Engineering, 7(6):707-721, 1983a. ISSN 00981354. doi: 10.1016/0098-1354(83)85023-6.

Papoulias, S. A. and Grossmann, I. E. A structural optimization approach in process synthesis-iii. Computers $\mathcal{E}$ Chemical Engineering, 7(6):723-734, 1983b. ISSN 00981354. doi: 10.1016/0098-1354(83)85024-8.

Peschel, A., Freund, H., and Sundmacher, K. Methodology for the Design of Optimal Chemical Reactors Based on the Concept of Elementary Process Functions. Industrial \& Engineering Chemistry Research, 49(21):10535-10548, 2010. ISSN 0888-5885. doi: 10.1021/ie100476q.

Pichardo, P. and Manousiouthakis, V. I. Infinite dimensional state-space as a systematic process intensification tool: Energetic intensification of hydrogen production. Chemical Engineering Research and Design, 120:372-395, 2017. ISSN 02638762. doi: 10.1016/j.cherd.2017.01.026. 
Rihko-Struckmann, L. K., Peschel, A., Hanke-Rauschenbach, R., and Sundmacher, K. Assessment of Methanol Synthesis Utilizing Exhaust $\mathrm{CO} 2$ for Chemical Storage of Electrical Energy. Industrial \& Engineering Chemistry Research, 49(21):11073-11078, 2010. ISSN 0888-5885. doi: 10.1021/ie100508w.

Ryu, J. and Maravelias, C. T. Simultaneous Process and Heat Exchanger Network Synthesis Using a Discrete Temperature Grid. Industrial \& Engineering Chemistry Research, 58(15):6002-6016, 2019. doi: 10.1021/acs.iecr.8b04083.

Schack, D. and Sundmacher, K. Techno-ökonomische Optimierung des Produktionsnetzwerkes für die Synthese von Ameisensäure aus erneuerbaren Ressourcen. Chemie Ingenieur Technik, 90(1-2):256-266, 2018. ISSN 0009286X. doi: 10.1002/cite.201700163.

Schack, D., Rihko-Struckmann, L., and Sundmacher, K. Structure optimization of power-to-chemicals (P2C) networks by linear programming for the economic utilization of renewable surplus energy. Computer Aided Chemical Engineering, 38:1551-1556, 2016. ISSN 1570-7946. doi: http://dx.doi.org/10.1016/ B978-0-444-63428-3.50263-0.

Schack, D., Rihko-Struckmann, L., and Sundmacher, K. Economic linear objective function approach for structure optimization of renewables-to-chemicals (R2Chem) networks. Computer Aided Chemical Engineering, 40:1975-1980, 2017.

Schack, D., Rihko-Struckmann, L., and Sundmacher, K. Linear Programming Approach for Structure Optimization of Renewable-to-Chemicals (R2Chem) Production Networks. Industrial 8 Engineering Chemistry Research, 57(30):9889-9902, 2018. ISSN 0888-5885. doi: 10.1021/acs.iecr.7b05305.

Schack, D., Liesche, G., and Sundmacher, K. Simultaneous Heat and Mass Flow Optimization of a Distillation Column Applying the FluxMax Approach. Chemical Engineering Transactions, 76:337-342, 2019.

Short, M., Isafiade, A. J., Biegler, L. T., and Kravanja, Z. Synthesis of mass exchanger networks in a two-step hybrid optimization strategy. Chemical Engineering Science, 178:118-135, 2018. doi: 10.1016/ j.ces.2017.12.019.

Surya Prakash, G. K., Olah, G. A., and Goeppert, A. Beyond oil and gas: The methanol economy. In ECS Transactions, volume 35, pages 31-40. doi: 10.1149/1.3645178.

Uebbing, J., Rihko-Struckmann, L. K., and Sundmacher, K. Exergetic assessment of CO2 methanation processes for the chemical storage of renewable energies. Applied Energy, 233-234:271 - 282, 2019. ISSN 0306-2619. doi: https://doi.org/10.1016/j.apenergy.2018.10.014.

Ulonska, K., Skiborowski, M., Mitsos, A., and Viell, J. Early-stage evaluation of biorefinery processing pathways using process network flux analysis. AIChE Journal, 62(9):3096-3108, 2016. ISSN 00011541. doi: 10.1002/aic.15305.

Voll, A. and Marquardt, W. Reaction network flux analysis: Optimization-based evaluation of reaction pathways for biorenewables processing. AIChE Journal, 58(6):1788-1801, 2012. ISSN 00011541. doi: 
$10.1002 /$ aic. 12704 .

Wilson, S. and Manousiouthakis, V. Ideas approach to process network synthesis: Application to multicomponent men. AIChE Journal, 46(12):2408-2416, 2000. ISSN 0001-1541. doi: 10.1002/aic.690461209.

Yee, T. F., Grossmann, I. E., and Kravanja, Z. Simultaneous optimization models for heat integration - iii. process and heat exchanger network optimization. Computers \& Chemical Engineering, 14(11):1185-1200, 1990. ISSN 00981354.

Yu, H., Eason, J., Biegler, L. T., and Feng, X. Simultaneous heat integration and techno-economic optimization of Organic Rankine Cycle (ORC) for multiple waste heat stream recovery. Energy, 119:322-333, 2017. ISSN 03605442. doi: 10.1016/j.energy.2016.12.061.

Zondervan, E., Nawaz, M., de Haan, A. B., Woodley, J. M., and Gani, R. Optimal design of a multi-product biorefinery system. Computers \& Chemical Engineering, 35(9):1752-1766, 2011. ISSN 00981354. doi: 10.1016/j.compchemeng.2011.01.042. 